\title{
HOMOGENEITY FOR OPEN PARTITIONS OF PAIRS OF REALS
}

\author{
QI FENG
}

\begin{abstract}
We prove a partition theorem for analytic sets of reals, namely, if $A \subseteq \mathbb{R}$ is analytic and $[A]^{2}=K_{0} \cup K_{1}$ with $K_{0}$ relatively open, then either there is a perfect 0 -homogeneous subset or $A$ is a countable union of 1 homogeneous subsets. We also show that such a partition property for coanalytic sets is the same as that each uncountable coanalytic set contains a perfect subset. A two person game for this partition property is also studied. There are some applications of such partition properties.
\end{abstract}

We study the existence of homogeneous sets for open partitions of pairs of reals with various underlying sets. In particular, we show that when the set of (unordered) pairs from an analytic subset of the reals is partitioned into two pieces so that one of them is relatively open, there is a strong form of homogeneity. From that, we derive some well-known theorems by specialization.

In giving a counterexample to generalizations of Ramsey's theorem [16], which says that if the square of the set of natural numbers is partitioned into two pieces then there must be an infinite subset whose square is contained in one of the pieces, Sierpiński [17] defined a partition of the plane by comparing a well order of the reals and the standard linear order of the reals such that there is no uncountable subset whose square is contained in one of the two pieces. Sierpinski's partition explicitly uses the well-orderability of the set of the reals which is highly noneffective. So a natural question would be what structural properties of a partition could give us desired homogeneity. A first positive result was proved by Galvin [8] (and later a more general result was given by Blass [3]), which says that if the square of the Cantor space is partitioned into two pieces satisfying that each piece is open then there is an uncountable (perfect) homogeneous set, i.e., whose square is contained in one of the pieces. (Precisely, Galvin proved a theorem for partitions of pairs or triples and conjectured a general theorem which Blass proved in [3]. But Galvin never published his arguments. In this paper, we will only consider partitions of pairs. So, by "the Blass-Galvin theorem" we mean the special case mentioned above. Interested readers can find the general theorem in [3].) Although the Blass-Galvin theorem gives us a very good answer to the question above, it is not as powerful as was expected.

Received by the editors April 13, 1990 and, in revised form, July 3, 1991.

1980 Mathematics Subject Classification (1985 Revision). Primary 03E05, 03E15, 03 E60.

Key words and phrases. Homogeneity, open-coloring, games, set theory, descriptive set theory.

This work was done during the year 1989-1990 when the author was visiting the Department of Mathematics at UC Berkeley. The author would like to thank the department for its hospitality during his visit. 
Abstracting from many applications, which most of the time are shown to be independent of the axioms of set theory (see also a paper by Abraham, Rubin, and Shelah [1] for related but weaker forms of partition statements), Todorcevic introduced the following Open-Coloring-Axiom (OCA) in [20]:

If $X \subseteq \mathbb{R}$ is uncountable, and $[X]^{2}=K_{0} \cup K_{1}$ is a partition with $K_{0}$ open in the relative topology, then either there is an uncountable 0-homogeneous subset, i.e., there is some uncountable $A \subseteq X$ with $[A]^{2} \subseteq K_{0}$, or $X$ is a countable union of 1-homogeneous sets, i.e., $X=\bigcup_{n<\omega} A_{n}$ with each $\left[A_{n}\right]^{2} \subseteq K_{1}$, where

$$
[X]^{2}=\{(x, y) \mid x, y \in X \wedge x<y\} .
$$

Let $\Gamma^{*}$ be the family of all the subsets $X \subseteq \mathbb{R}$ satisfying the property specified in the OCA above for each open partition. A natural question here would be which uncountable subsets of the reals are in $\Gamma^{*}$. OCA claims exactly that $\Gamma^{*}=$ the power set of $\mathbb{R}$.

It is shown in [20] that OCA is consistent with the axioms of set theory. By the works of Todorcevic [20,22] and Velickovic [23], OCA has many interesting consequences. Clearly, the homogeneity given by OCA is something we would like very much to have.

Let $\Gamma$ be a family of subsets of the reals. Let us consider the following statement $\operatorname{OCA}(\Gamma)$ :

For each $X \in \Gamma$, if $[X]^{2}=K_{0} \cup K_{1}$ is a partition with $K_{0}$ open in the relative topology, then either there is a perfect subset $P \subseteq X$ which is 0-homogeneous, i.e., $[P]^{2} \subseteq K_{0}$, or $X$ is a countable union of 1-homogeneous sets, i.e., $X=$ $\bigcup_{n=1}^{\infty} A_{n}$ with $\left[A_{n}\right]^{2} \subseteq K_{1}$ for each positive integer $n$.

If $\Gamma$ is a singleton $\{X\}$, let $\operatorname{OCA}(X)$ denote $\operatorname{OCA}(\{X\})$.

Let $\Gamma^{* *}=\{X \subseteq \mathbb{R} \mid \operatorname{OCA}(X)\}$.

Now a more interesting question is which $X \subseteq \mathbb{R}$ are in $\Gamma^{* *}$. Trivially all countable subsets of the reals are in $\Gamma^{* *}$. Let us also point out that if the Axiom of Choice is assumed, then $\Gamma^{* *}$ cannot be the family of all subsets of the reals. Actually, in $\S 1$ we will see that, assuming the axiom of choice, $\Gamma^{* *} \neq \Gamma^{*}$.

First let us notice that both $\Gamma^{* *}$ and $\Gamma^{*}$ have some nice closure properties, namely, they are closed under countable unions, closed under continuous images, and invariant modulo the $\sigma$-ideal of the countable subsets of the reals. More precisely, we summarize these properties in the following lemma, leaving the easy proof to the reader.

Lemma. (1) If for each $n \operatorname{OCA}\left(X_{n}\right)$ holds, then $\mathrm{OCA}\left(\cup_{n=1}^{\infty} X_{n}\right)$.

(2) If $\operatorname{OCA}(X)$ holds, $f: X \rightarrow \mathbb{R}$ is continuous, then $\operatorname{OCA}(f[X])$, where $f[X]=\{f(a) \mid a \in X\}=f^{\prime \prime} X$.

(3) If $\operatorname{OCA}(X)$ holds, $Y \subseteq \mathbb{R}$, and $|X \triangle Y| \leq \aleph_{0}$, then $\operatorname{OCA}(Y)$, where $X \triangle Y=(X-Y) \cup(Y-X)$.

This paper is organized as follows.

In $\S 1$, we show that if $\Gamma$ is the family of the analytic sets, then $\operatorname{OCA}(\Gamma)$ holds. Hence the Blass-Galvin theorem follows. So does the classical perfect set theorem of Souslin. Also it follows that every Bernstein set is in $\Gamma^{*}$. An example of Todorcevic [21] is included which combined with the results of $\S 2$ shows that this theorem is the best possible.

In $\S 2$, we consider the family of all coanalytic sets, denoted by $\underline{I}_{1}^{1}$. It turns out that $\operatorname{OCA}\left(\underline{\Pi}_{1}^{1}\right)$ is equivalent to that every uncountable coanalytic subset of 
the reals contains a perfect subset. What we actually show is a stronger form of the Mansfield-Solovay Perfect Set Theorem, which will follow as a special case.

In $\S 3$, we define a two person game associated with a given open partition. By doing that we show, for example, if $\Gamma$ is the family of all projective sets, then $\operatorname{OCA}(\Gamma)$ follows from the Projective Determinacy Hypothesis (PD). Also playing such a game gives another proof that every analytic subset is in $\Gamma^{* *}$.

In $\S 4$, we show that OCA holds in Solovay's models.

In $\S 5$, we list some applications.

In $\S 6$, we generalize a partition theorem of Erdös and Rado to the context where the reals are not assumed to be well-orderable.

In the rest of this introduction, we fix some notations.

Notice that it follows from the lemma above that, for $\operatorname{OCA}(\Gamma)$, it does not matter whether we consider the whole real line, the set of irrational numbers, the unit interval, or the Cantor space. So we will work on any of the spaces whenever it is convenient. Also we could consider in general any Polish space, not only the reals.

We use $\omega$ to denote the set of all nonnegative integers. Given a set $X$, we use $P(X)$ to denote the family of all subsets of $X$. By $X^{<\omega}$ we denote the set of all finite sequences of elements of $X$. Fix $s, t \in X^{<\omega} \cdot \ln (s)$ is the length of $s$, i.e., $s: \ln (s) \rightarrow X$. We use $t \leq s$ to mean that $s$ is an initial segment of $t$, i.e, there is a positive integer $n \leq \operatorname{lh}(t)$ such that $s=t\lceil n$, where $t \uparrow n$ is the restriction of $t$ to $n$. By $s \perp t$, we mean that $s$ is incompatible with $t$, i.e., there is some positive integer $n<\min (\operatorname{lh}(s), \operatorname{lh}(t))$ such that $s(n) \neq t(n)$. A subset $T \subseteq X^{<\omega}$ is a tree on $X$ if $T$ is closed under initial segments. (Our trees will grow downward.) Given a tree $T \subseteq X^{<\omega}$, by [T] we denote the set of all branches through $T$, i.e., all functions $f: \omega \rightarrow X$ such that for each $n, f \mid n \in T$. If $T \subseteq X^{<\omega}$ is a tree, and $s \in T$, by $T_{s}$ we denote the following subtree $T_{s}=\{t \in T \mid t \leq s \vee s \leq t\}$.

If $X=A \times B, T \subseteq X^{<\omega}$ is a tree, we usually identify $T$ with

$$
\{(s, t) \mid \exists h \in T \operatorname{lh}(s)=\operatorname{lh}(t)=\operatorname{lh}(h) \wedge h(i)=(s(i), t(i)) \text { for all } i\} .
$$

Then by $p[T]$ we denote the projection of the tree $T$ in this situation, which is the set

$$
p[T]=\{g \mid g: \omega \rightarrow A \wedge \exists f: \omega \rightarrow B \forall n(g|n, f| n) \in T\} .
$$

By $\omega^{\omega}$ we denote the Baire space, i.e., the product space of $\omega$ with $\omega$ equipped with the discrete topology, which is a complete separable metric space homeomorphic to the irrationals. Similarly we use $2^{\omega}$ to denote the product space homeomorphic to the Cantor space. We will consider the reals to be the Baire space most of the time with the exception of $\S 3$ where we consider the Cantor space. Sometimes, we consider the products of them.

For $s \in \omega^{<\omega}$, we use $N_{s}$ to denote the basic open (clopen) set determined by $s$, i.e., $N_{s}=\left\{f \in \omega^{\omega} \mid f \uparrow \operatorname{lh}(s)=s\right\}$.

Similarly, for $s \in 2^{<\omega}$, we use $N_{s}$ to denote the open set determined by $s$ in the Cantor space.

Let $\mathbb{R}$ be the set of reals, which could be one of the spaces mentioned above. For $X \subseteq \mathbb{R}$,

$$
[X]^{2}=\{(x, y) \mid x, y \in X, x<y\}
$$


which we sometimes identify with

$$
[X]^{2}=\{\{x, y\} \mid x, y \in X \wedge x \neq y\} .
$$

By the topology on $[\mathbb{R}]^{2}$ we mean the subspace topology induced from $\mathbb{R} \times \mathbb{R}$.

A nonempty subset $X \subseteq \mathbb{R}$ is analytic if there is a continuous function $f$ from $\omega^{\omega}$ to $\mathbb{R}$ such that $X=f\left[\omega^{\omega}\right] . X \subseteq \mathbb{R}$ is coanalytic if the complement of $X$ is analytic. We use $\underline{\Sigma}_{1}^{1}$ to denote the family of all analytic sets, $\underline{I}_{1}^{1}$ to denote the family of all coanalytic sets. Notice that all Borel sets are both analytic and coanalytic. We also use $\Sigma_{2}^{1}$ to denote the family of all subsets of the reals which are continuous images of coanalytic subsets of $\omega^{\omega}$. By projective sets we mean those which can be obtained by finite iteration of taking continuous images and complementations. I.e., inductively, let $\underline{\boldsymbol{I}}_{n}^{1}$ be the family of all complements of $\underline{\Sigma}_{n}^{1}$ sets, and let $\underline{\Sigma}_{n+1}^{1}$ be the family of all continuous images of the $\underline{\boldsymbol{I}}_{n}^{1}$ sets. Then the family of the projective sets is the union of all of them. By the lemma, to show $\operatorname{OCA}\left(\underline{\Sigma}_{1}^{1}\right)$ it suffices to show $\operatorname{OCA}(\mathbb{R})$, and $\operatorname{OCA}\left(\underline{\boldsymbol{\Pi}}_{n}^{1}\right)$ implies $\operatorname{OCA}\left(\underline{\boldsymbol{\Sigma}}_{n+1}^{1}\right)$.

We use ZF to denote the Zermelo-Fraenkel axioms of set theory. By DC we mean the Dependent Choice Principle: if $R$ is a binary relation on a nonempty set $X$, and if for every $a \in X$ there is $a b \in X$ such that $R(a, b)$, then there is an infinite sequence $\left\langle a_{n} \mid n<\omega\right\rangle$ from $X$ satisfying $R\left(a_{n}, a_{n+1}\right)$ for each $n<\omega$. By AC, we mean the Axiom of Choice.

We shall need the following two basic facts, which can be found in many text books in descriptive set theory or, e.g., in Jech [10].

Fact 1. Let $X$ be either $2, \omega$ or $\omega \times \omega$. Then for any $C \subseteq X^{\omega}, C$ is closed if and only if there is a tree $T \subseteq X^{<\omega}$ such that $C=[T]$.

Fact 2. $X \subseteq \mathbb{R}$ is analytic if and only if there is a closed set $C \subseteq \mathbb{R} \times \omega^{\omega}$ such that $X=\{x \mid \exists y(x, y) \in C\}$ if and only if there is a tree $T$ on $\omega \times \omega$ such that $X=p[T]$.

To end this introduction, let us record our acknowledgments. This work was done after the two motivating talks of Todorcevic [22] and Velickovic [23] at the MSRI Workshop on Set Theory in Berkeley, October 1989. I would like to thank S. Todorcevic for many stimulating discussions, many times on the bus riding on the road between the Institute and the Berkeley mathematics department or in the Coffee Connection (1807 Euclid Ave), and for many valuable suggestions, corrections in writing this paper, which makes the paper different in many places from the first draft; I acknowledge here my debt to him. I would also like to thank B. Velickovic for many interesting conversations on the subject of this paper. I especially thank Professor Hugh Woodin for financial support in the year of 1989 and for many interesting conversations on the subject. I would like also to thank L. Harrington, T. Jech, W. Just, A. Kechris, M. Magidor, D. A. Martin, A. Mathias, S. Simpson, and R. Solovay for their interest and encouragement. A special thanks is due to the referee for a long and detailed report which helped me to correct many of my English errors and to polish my presentation. 


\section{OPEN COLORING OF PAIRS FROM ANALYTIC SETS}

In this section, we show in the theory $\mathrm{ZF}$ that $\operatorname{OCA}\left(\boldsymbol{\Sigma}_{1}^{1}\right)$ holds. Then we deduce the classical perfect set theorem and the Blass-Galvin partition theorem (for the pairs). We also include a partition of Todorcevic from [21] which shows that the dual version of $\operatorname{OCA}\left(\underline{\Sigma}_{1}^{1}\right)$ is not true. The proof of $\operatorname{OCA}\left(\underline{\Sigma}_{1}^{1}\right)$ to be given is in some sense similar to the Cantor-Bendixson analysis for the closed sets in the Baire space.

Theorem 1.1 (ZF). OCA $\left(\underline{\Sigma}_{1}^{1}\right)$. That is, if $A \subseteq \mathbb{R}$ is analytic, and $[A]^{2}=K_{0} \cup K_{1}$ is a partition with $K_{0}$ relatively open, then either there is a perfect subset which is 0-homogeneous or $A$ is a countable union of 1-homogeneous sets (i.e., $A=$ $\bigcup_{n<\omega} X_{n}$ and for each $\left.n<\omega,\left[X_{n}\right]^{2} \subseteq K_{1}\right)$.

Before we get into the proof, let us point out the following fact. Given a partition $[A]^{2}=K_{0} \cup K_{1}$ with $K_{0}$ relatively open, if $A$ is a countable union of 1-homogeneous sets, then $A$ is a countable union of relatively closed 1homogeneous sets. Namely, if $B \subseteq A$ is 1-homogeneous, if $C$ is the closure of $B$, then $C \cap A$ is 1-homogeneous.

Proof. Let $A$ be analytic. Let $T \subseteq \omega^{<\omega} \times \omega^{<\omega}$ be a tree such that $A=p[T]$.

Assume that $A$ is not a countable union of 1-homogeneous sets. We are going to construct a perfect subset which is 0-homogeneous.

By Shoenfield absoluteness [18], we could carry out the following arguments inside some inner model $L[a]$ for some real $a$. Therefore, we may assume without loss of generality that DC holds.

For $(s, t) \in T$, define that $(s, t) \in T^{\prime}$ if and only if $p\left[T_{(s, t)}\right]$ is not a countable union of 1-homogeneous sets, where $T_{(s, t)}=\left\{\left(s^{\prime}, t^{\prime}\right) \in T \mid\left(s^{\prime}, t^{\prime}\right) \leq\right.$ $(s, t)$ or $\left.(s, t) \leq\left(s^{\prime}, t^{\prime}\right)\right\}$. For $(s, t) \in T^{\prime}$, let $T_{(s, t)}^{\prime}=T^{\prime} \cap T_{(s, t)}$.

Claim 1. If $(s, t) \in T^{\prime}$, then $p\left[T_{(s, t)}^{\prime}\right]$ is not a countable union of 1-homogeneous sets.

Assume otherwise. Let $(s, t) \in T^{\prime}$ be such that $p\left[T_{(s, t)}^{\prime}\right]$ is a countable union of 1-homogeneous sets. For $(\sigma, \tau) \in T_{(s, t)}-T^{\prime}, p\left[T_{(\sigma, \tau)}\right]$ is a countable union of 1-homogeneous sets. For $x \in p\left[T_{(s, t)}\right]$, either $x \in p\left[T_{(s, t)}^{\prime}\right]$ or there is some $(\sigma, \tau) \in T_{(s, t)}-T^{\prime}$ such that $x \in p\left[T_{(\sigma, \tau)}\right]$. Hence $p\left[T_{(s, t)}\right]$ is a countable union of 1 -homogeneous sets. This is a contradiction.

Claim 2. If $(s, t) \in T^{\prime}$, then there are $\left(s_{0}, t_{0}\right) \in T_{(s, t)}^{\prime},\left(s_{1}, t_{1}\right) \in T_{(s, t)}^{\prime}$ such that $s_{0}$ and $s_{1}$ are incompatible and $p\left[T_{\left(s_{0}, t_{0}\right)}\right] \times p\left[T_{\left(s_{1}, t_{1}\right)} \subseteq K_{0}\right.$.

To see this, let $(s, t) \in T^{\prime}$. Since $p\left[T_{(s, t)}^{\prime}\right]$ is not a countable union of 1homogeneous sets, there are distinct $x_{0}, x_{1} \in p\left[T_{(s, t)}^{\prime}\right]$ such that $\left\{x_{0}, x_{1}\right\} \in K_{0}$. Since $K_{0}$ is open, and $x_{0} \neq x_{1}$, we can find some large $n$ such that $x_{0}\lceil n$ is incompatible with $x_{1} \mid n$ and for all $x, y$ with $x\left|n=x_{0}\right| n$ and $y\left|n=x_{1}\right| n$ if both $x \in A$ and $y \in A$ then $\{x, y\} \in K_{0}$.

Let $s_{0}=x_{0} \mid n$ and $s_{1}=x_{1} \mid n$. Since $x_{0}, x_{1} \in p\left[T_{(s, t)}^{\prime}\right]$, there are $t_{0}$, $t_{1}$ such that $\left(s_{0}, t_{0}\right) \in T_{(s, t)}^{\prime}$ and $\left(s_{1}, t_{1}\right) \in T_{(s, t)}^{\prime}$. Then $p\left[T_{\left(s_{0}, t_{0}\right)}\right] \times p\left[T_{\left(s_{1}, t_{1}\right)}\right]$ $\subseteq K_{0}$. 
Now, since $T^{\prime}$ is not empty, we can construct by induction a perfect tree $T^{*} \subseteq \omega^{<\omega}$ such that $\left[T^{*}\right] \subseteq p[T]$ and $\left[\left[T^{*}\right]\right]^{2} \subseteq K_{0}$.

Remark. Similar arguments show that if $A \subseteq \mathbb{R}$ is $\kappa$-Souslin, and $[A]^{2}=K_{0} \cup$ $K_{1}$ with $K_{0}$ open, then either there is a perfect 0-homogeneous subset or $A$ is the union of $\kappa$ 1-homogeneous sets. In particular, for every ${\underset{\Sigma}{2}}_{2}^{1}$ subset $A \subseteq \mathbb{R}$, if $[A]^{2}=K_{0} \cup K_{1}$ with $K_{0}$ open, then either there is a perfect subset which is 0-homogeneous or $A=\bigcup_{\alpha<\omega_{1}} X_{\alpha}$ with each $\left[X_{\alpha}\right]^{2} \subseteq K_{1}$, where $\omega_{1}$ is the first uncountable cardinal. On the other hand, in $\mathrm{ZF}+\mathrm{AC}$, one cannot prove that $\operatorname{OCA}\left(\underline{\Pi}_{1}^{1}\right)$, since there may be some uncountable coanalytic sets not containing a perfect subset in some universe of set theory, while $\operatorname{OCA}\left(\underline{\Pi}_{1}^{1}\right)$ implies that every coanalytic set is either countable or contains a perfect subset. Todorcevic shows in [20] that assuming Gödel's $V=L$, there is an uncountable $\underline{I}_{1}^{1}$ set $A$ and an open partition of $[A]^{2}$ such that there are no uncountable homogeneous sets of either color. Namely, by Erdös, Kunen, and Mauldin [25], assuming $V=L$, there is a $\Pi_{1}^{1}$ scale, i.e., there is a $\Pi_{1}^{1}$ sequence $X=\left\{f_{\alpha} \mid \alpha<\omega_{1}\right\}$ such that each $f_{\alpha} \in \omega^{\omega}$ is increasing, if $\alpha<\beta$ then $\exists n \forall m \geq n f_{\alpha}(m)<f_{\beta}(m)$, (i.e., $X$ is $\Pi_{1}^{1}$ and every member of $X$ is increasing and $X$ is well ordered by the ordering of eventual dominance with order type $\omega_{1}$ ), and for each $f \in \omega^{\omega}$ there is some $\alpha$ such that $f$ is eventually dominated by $f_{\alpha}$. Now the partition $[X]^{2}=K_{0} \cup K_{1}$ defined by $\alpha<\beta$,

$$
\left\{f_{\alpha}, f_{\beta}\right\} \in K_{0} \Leftrightarrow \exists m f_{\alpha}(m)>f_{\beta}(m)
$$

is the desired partition (see [20, p. 62] for a proof). We will get back to this in the next section to see why $V=L$ is needed.

Let us see some interesting consequences of Theorem 1.1.

Lemma 1.1. For $X \subseteq \mathbb{R}, \operatorname{OCA}(X)$ implies that $X$ either is countable or contains a perfect subset.

Proof. Consider the trivial partition $[X]^{2}=K_{0} \cup K_{1}$ with $K_{1}=\varnothing$.

Corollary 1.1 (Souslin). Every uncountable analytic set contains a perfect subset.

Corollary 1.2 (Blass-Galvin). If $[\mathbb{R}]^{2}=K_{0} \cup K_{1}$ is a partition with $K_{0}$ analytic, then there is a perfect subset $P$ which is homogeneous, i.e., either $[P]^{2} \subseteq K_{0}$, or $[P]^{2} \subseteq K_{1}$.

Proof. Let $A, B$ be two open sets such that $K_{0} \triangle A$ and $B \triangle K_{1}$ are meager. By Mycielski's theorem [15], there is a perfect set $D \subseteq \mathbb{R}$ such that $[D]^{2} \subseteq$ $\left(A \cap K_{0}\right) \cup\left(B \cap K_{1}\right)$. By the theorem above, we get either there is a perfect subset which is 0 -homogeneous, in which case we are done, or $D$ is a countable union of closed sets which are 1-homogeneous. So one of them is uncountable, hence contains a perfect subset.

In the following, we would like to point out how Theorem 1.1 helps us in understanding the Open-Coloring-Axiom (OCA) assuming the axiom of choice. Namely, OCA essentially is a statement asserting all singular subsets of the reals have a certain regularity property.

As for the regularity properties of the subsets of the reals, the following theorem seems very interesting. 
Theorem 1.2. Let $X \subseteq \mathbb{R}$. Then either $\mathbb{R}-X$ contains a perfect subset or $X \in \Gamma^{*}$.

Proof. Assume that $X \notin \Gamma^{*}$. Then there must be an open partition $[X]^{2}=$ $K_{0} \cup K_{1}$ such that for some $A \subseteq[\mathbb{R}]^{2} A$ is open, $K_{0}=A \cap[X]^{2}$, and there are no uncountable 0 -homogeneous sets and $X$ is not a countable union of 1 -homogeneous sets. Look at the open partition of $[\mathbb{R}]^{2}$ given by $A$. Clearly $\mathbb{R}$ is not a countable union of 1-homogeneous sets, as otherwise $X$ would be. So by Theorem 1.1, there must be a perfect $P \subseteq \mathbb{R}$ such that $[P]^{2} \subseteq A$. Since $X$ contains no uncountable 0 -homogeneous subset, $P \cap X$ must be countable. Hence $P-X$ contains a perfect subset.

A subset $X \subseteq \mathbb{R}$ is a Bernstein set if both $X$ and $\mathbb{R}-X$ doe not contain a perfect subset. A subset $X \subseteq \mathbb{R}$ is an $S_{0}$-set if for every perfect set $P$ there is a perfect subset $Q \subseteq P$ such that $X \cap Q=\varnothing$. We have the following corollary immediately.

Corollary 1.3. (1) If $X$ is Bernstein, then $X \in \Gamma^{*}$.

(2) If $X$ is an $S_{0}$-set, then $\mathbb{R}-X \in \Gamma^{*}$.

It follows that all the complements of the following singular subsets of the real line have the OCA property: Luzin sets, Sierpiński sets, concentrated sets, $C^{\prime \prime}$-sets, $C^{\prime}$-sets, strong measure zero sets, universal measure zero sets, $Q$-sets, $\sigma$-sets, $\lambda$-sets, $\lambda^{\prime}$-sets, perfect meager sets (see, e.g., [28] for definitions). Since $A C$ implies that Bernstein sets exist, $A C$ implies that $\Gamma^{* *} \neq \Gamma^{*}$.

We will have more applications in $\S 5$. To end this section, let us reproduce an example of Todorcevic from [21] for completeness.

For each real $f: \omega \rightarrow \omega$, we associate a sequence $\left\langle f_{n}: n<\omega\right\rangle$ converging to $f$ as follows.

We define $f_{i}\left\lceil i=f\left\lceil i\right.\right.$ and $f_{i}(i+j)=f\left(2^{i+1}(2 j+1)\right)$.

Now we define the partition as $[\mathbb{R}]^{2}=K_{0} \cup K_{1}$, where $\{f, g\} \in K_{0} \Leftrightarrow \forall i f \neq$ $g_{i} \& g \neq f_{i}$.

Claim $1 . K_{0}$ is open.

To see this, let $f, g$ be two reals such that $f \neq g$ and for each $i$ we have $f \neq g_{i}$. Let $m$ be such that $f \backslash m=g \backslash m$ and $f(m) \neq g(m)$. Pick $m^{*}$ such that $\forall i \leq m \exists j<m^{*} f(j) \neq g_{i}(j)$. Then let

$$
m^{* *}=2^{m+1}\left(2 m^{*}+1\right)+1 .
$$

Subclaim. If $g^{\prime}\left|m^{* *}=g\right| m^{* *}, f^{\prime} \mid m^{*}=f\left\lceil m^{*}\right.$, then $\forall i f^{\prime} \neq g_{i}^{\prime}$.

By symmetry, Claim 1 follows from the Subclaim.

We need to check the Subclaim. First, for $i \leq m, g_{i}^{\prime} \backslash m^{*}=g_{i} \backslash m^{*}$.

Assume that $f^{\prime}=g_{i}^{\prime}$ for some $i$. Then $f^{\prime}\left|i=g^{\prime}\right| i$. Since $f^{\prime}(m) \neq$ $g^{\prime}(m)$, we must have $i \leq m$. But then there is some $j<m^{*}$ such that $f^{\prime}(j)=f(j) \neq\left(g_{i}(j)=g_{i}^{\prime}(j)\right.$.

Claim 2. The set of reals is not a countable union of 0-homogeneous sets.

Let $\left\{H_{i} \mid i<\omega\right\}$ be a countable set of 0 -homogeneous sets. We want to find a real $f$ not in any of the $H_{i}$.

Pick $f_{0} \in H_{0}$. Define $f(2(2 j+1))=f_{0}(j)$. Pick $f_{1} \in H_{1}$. Define $f(0)=$ $f_{1}(0)$ and $f(4(2 j+1))=f_{1}(1+j)$. Then let $s_{0}=f \nmid 1$.

Let $N_{0}=\left\{g \mid s_{0} \subseteq g\right\}$. 
If $H_{2} \cap N_{s_{0}}=\varnothing$, then define $f(1)=f(0)+1$ and define $f\left(2^{3}(2 j+1)\right)$ arbitrarily. If otherwise, let $f_{2} \in H_{2} \cap N_{0}$. Then define $f(1)=f_{2}(1)$ and $f\left(2^{3}(2 j+1)\right)=f_{2}(2+j)$. In either case, let $s_{1}=f \nmid 3$, and let $N_{1}=\left\{g \mid s_{1} \subseteq g\right\}$.

If $H_{3} \cap N_{1}$ is not empty, let $f_{3} \in H_{3} \cap N_{1}$. Then define $f(3)=f_{3}(3)+1$ and $f\left(2^{3}(2 j+1)\right)=f_{3}(3+j)$. If $H_{3} \cap N_{1}$ is empty, then define $f(3)=f(1)+1$ and $f\left(2^{3}(2 j+1)\right)$ arbitrarily. In any case, let $s_{2}=f \mid 5$.

Continue this way for $\omega$ many steps. We claim that the $f$ defined is not in any of the $H_{i}$. Given $i \geq 2$, if $H_{i} \cap N_{i-2}$ is empty, then trivially $f \notin H_{i}$. If the intersection is not empty or $i<2$, then $f_{i} \in H_{i}$ and $f_{i}$ is the $i$ th member of the sequence associated with $f$. Hence $\left\{f, f_{i}\right\} \in K_{1}$, which implies $f \notin H_{i}$.

Claim 3. If $X$ is an uncountable subset of the reals, then there are two reals $f, g$ in $X$ so that $\{f, g\} \in K_{0}$.

Let $\left\{f^{\alpha} \mid \alpha<\omega_{1}\right\}$ be an uncountable set of reals. For each $\beta<\omega_{1}$, the set $\left\{f_{i}^{\alpha} \mid \alpha<\beta, i<\omega\right\}$ is countable. So by passing to an uncountable subset if necessary, we may assume that for each $\beta$, for each $\alpha<\beta$ and for each $i<\omega$ we have that $f^{\beta} \neq f_{i}^{\alpha}$.

Since $\left\{f_{i}^{\omega} \mid i<\omega\right\} \cap\left\{f_{i}^{\omega+1} \mid i<\omega\right\}$ is finite, there are some $n<\omega$ and some $\alpha \in\{\omega, \omega+1\}$ such that $f^{n} \neq f_{i}^{\alpha}$ for each $i<\omega$. Therefore, $\left\{f^{n}, f^{\alpha}\right\} \in K_{0}$ for such a pair $(n, \alpha)$.

\section{OPEN COLORING OF PAIRS FROM COANALYTIC SETS}

As we promised in $\S 1$, we consider the situation of $\operatorname{OCA}\left(\underline{\Pi}_{1}^{1}\right)$. We show that $\operatorname{OCA}\left(\underline{\Pi}_{1}^{1}\right)$ is equivalent to that every $\underline{\Sigma}_{2}^{1}$ subset either is countable or contains a perfect subset. We will basically follow the Shoenfield [18], Mansfield and Solovay [12,19] analysis of the $\Sigma_{2}^{1}$ sets. By combining them, we show first a stronger form of the Mansfield and Solovay theorem. The proof will be essentially Solovay's proof plus Shoenfield absoluteness. So the proof will be similar to the Cantor-Bendixson analysis.

First let us recall the Shoenfield analysis of the $\Sigma_{2}^{1}$ sets. That is, a subset $X \subseteq \mathbb{R}$ is $\Sigma_{2}^{1}$ if and only if there is a $\Sigma_{2}^{1}$ formula $\varphi(x, y)$ of the analysis and some real number $a \in \mathbb{R}$ such that $X=\{b \in \mathbb{R} \mid \varphi(b, a)\}$. Therefore, if $X \subseteq \mathbb{R}$ is $\underline{\Sigma}_{2}^{1}$ then there is a tree $T$ on $\omega \times \omega_{1}$ such that $X=p[T]$. Furthermore, in case $X$ is $\underline{\Sigma}_{2}^{1}, \varphi(x, y)$ is a $\Sigma_{2}^{1}$ formula, $a \in \mathbb{R}$, and $X=\{b \in \mathbb{R} \mid \varphi(b, a)\}$, then we can choose a tree $T$ on $\omega \times \omega_{1}$ such that $X=p[T]$ and $T$ is constructible from the real $a$, which is important to us. Also in this case we say that $X$ is a $\Sigma_{2}^{1}(a)$ subset of the reals.

Given a real $a$, we use $L[a]$ to denote the Gödel constructible universe from the real $a$. For more about the universe $L[a]$, see Jech [10].

Let $\left\langle\tau_{n}\right| n\langle\omega\rangle$ be a recursive $1-1$ enumeration of the sequences in $(\omega \times \omega)^{<\omega}$. Given an open set $A \subseteq[\mathbb{R}]^{2}, A$ is a countable union of basic open sets. So we can have a real $f: \omega \rightarrow \omega$ such that $A=\bigcup_{n<\omega} N_{\tau_{f(n)}}$. We call such an $f$ a code of $A$.

Theorem 2.1. Let $X$ be a $\Sigma_{2}^{1}(a)$ subset of the reals. Let $A$ be an open subset of $[\mathbb{R}]^{2}$. Let $c$ be a code for $A$. Assume that $c \in L[a]$, and $[X]^{2}=K_{0} \cup K_{1}$ with $K_{0}=A \cap[X]^{2}$. Then either there is a perfect tree $F \in L[a]$ on $\omega$ such 
that $[[F]]^{2} \subseteq K_{0}$, or for each $y \in X$ there is a tree $F \in L[a]$ on $\omega$ such that $y \in[F]$ and $[[F] \cap X]^{2} \subseteq K_{1}$.

Proof. Let $T \in L[a]$ be a tree on $\omega \times \omega_{1}$ such that $X=p[T]$ and let $\varphi(x, y)$ be a $\Sigma_{2}^{1}$ formula such that for each $y \in \mathbb{R}$ we have that $y \in X \Leftrightarrow \varphi(y, a)$. Also let $c \in \omega^{\omega}$ be a code for $A$ such that $c \in L[a]$ and $[X]^{2}=K_{0} \cup K_{1}$ with $K_{0}=A \cap[X]^{2}$.

For a subtree $S \subseteq T$, we define the subtree $S^{\prime} \subseteq S$ as follows:

For $(s, t) \in S$, we let $(s, t) \in S^{\prime}$ if and only if there are $\left(s_{0}, t_{0}\right) \in S$ and $\left(s_{1}, t_{1}\right) \in S$ satisfying that $s \subseteq s_{0}, s \subseteq s_{1}, t \subseteq t_{0}, t \subseteq t_{1}, s_{0} \perp s_{1}$, and for each $x, y$ if $s_{0} \subseteq x, s_{1} \subseteq y$, and $\varphi(x, a) \wedge \varphi(y, a)$, then there is an $n<\omega$ such that $(x, y) \in N_{\tau_{c(n)}}$.

Notice that if $S \in L[a]$, then $S^{\prime} \in L[a]$ since

$$
\forall x \forall y s_{0} \subseteq x \wedge s_{1} \subseteq y \wedge \varphi(x, a) \wedge \varphi(y, a) \rightarrow \exists n<\omega(x, y) \in N_{\tau_{c(n)}}
$$

is a $\Pi_{2}^{1}(a, c)$ statement, hence absolute with respect to $L[a]$ by Shoenfield absoluteness.

Now we work in $L[a]$. Inductively define $T^{(\alpha)}$ as follows:

$$
\begin{aligned}
& T^{(0)}=T . \\
& T^{(\alpha+1)}=\left(T^{(\alpha)}\right)^{\prime} \\
& T^{(\lambda)}=\bigcap_{\alpha<\lambda} T^{(\alpha)} \text { for limit } \lambda .
\end{aligned}
$$

We are now back to the universe, keeping in mind that the definition is absolute by the previous remark.

Let $\alpha$ be the least such that $T^{(\alpha+1)}=T^{(\alpha)}$.

Case 1. $T^{(\alpha)}=\varnothing$.

In this case, we claim that for each $y \in X$ there is a tree $F \in L[a]$ on $\omega$ such that $y \in[F]$ and $[[F] \cap X]^{2} \subseteq K_{1}$.

So fix $y \in X$. Let $f \in \omega_{1}^{\omega}$ be such that $(y, f) \in[T]$.

Let $\rho<\alpha$ be such that $(y, f) \in\left[T^{(\rho)}\right],(y, f) \notin\left[T^{(\rho+1)}\right]$. Let $(s, t) \in T^{(\rho)}$ be such that $s \subseteq y, t \subseteq f$ and $(s, t) \notin T^{(\rho+1)}$.

Subcase 1. $\forall\left(s^{\prime}, t^{\prime}\right) \in T^{(\rho)}\left(s^{\prime}, t^{\prime}\right) \leq(s, t) \Rightarrow s^{\prime} \subseteq y$.

Then we have $y=\bigcup\left\{s^{\prime} \leq s \mid \exists t^{\prime} \leq t\left(s^{\prime}, t^{\prime}\right) \in T^{(\rho)}\right\}$. Since the tree $T^{(\rho)} \in$ $L[a], y \in L[a]$. Now take the tree $F=\{y|n| n<\omega\}$.

Subcase 2. Otherwise.

In this case, we must have that there are $\left(s_{0}, t_{0}\right),\left(s_{1}, t_{1}\right) \in T^{(\rho)}$ such that $s_{0} \leq s, s_{1} \leq s, t_{0} \leq t, t_{1} \leq t$ and $s_{0} \perp s_{1}$. Also, for each such splitting pair $\left(s_{0}, t_{0}\right),\left(s_{1}, t_{1}\right)$, we have that

$$
\left[\left(N_{s_{0}} \cap X\right) \times\left(N_{s_{1}} \cap X\right)\right] \cap K_{1} \neq \varnothing .
$$

Let $S=T_{(s, t)}^{(\rho)}$. Then $S \in L[a]$.

Claim. $\left[N_{s} \cap p[S]\right]^{2} \subseteq K_{1}$.

Otherwise, let $d, b \in N_{s} \cap p[S]$ be such that $\{d, b\} \in K_{0}$. Since $K_{0}$ is open, there is an $m>\operatorname{lh}(s)$ such that $d\left\lceil m \neq b\left\lceil m\right.\right.$ and $\left(N_{d \backslash m} \cap X\right) \times\left(N_{b \backslash m} \cap\right.$ $X) \subseteq K_{0}$. Since $d, b \in p[S]$, there are $t_{0}, t_{1} \leq t$ such that $\left(d \backslash m, t_{0}\right)$, $\left(b \mid m, t_{1}\right) \in T^{(\rho)}$. Now by absoluteness, $(s, t) \in T^{(\rho+1)}$. Contradiction.

Notice that by absoluteness, the claim is true in $L[a]$. Now work in $L[a]$. Let $F$ be a tree on $\omega$ such that $[F]=$ the closure of $N_{s} \cap p[S]$. Since $K_{0}$ is 
open, we have that $[[F] \cap p[T]]^{2} \subseteq K_{1}$. Back in the universe, by absoluteness, we have the above inclusion holds. Furthermore, $y \in N_{s} \cap p[S] \subseteq[F]$.

Case 2. $T^{(\alpha)} \neq \varnothing$.

In this case, by induction, we define a perfect tree $F$ on $\omega$ so that

$$
F \in L[a] \wedge[F] \subseteq p\left[T^{(\alpha)}\right] \wedge[F]^{2} \subseteq K_{0} .
$$

To proceed, let $\left(s_{\varnothing}, t_{\varnothing}\right) \in T^{(\alpha)}$.

Let $\sigma \in 2^{<\omega}$. Inductively assume that $\left(s_{\sigma}, t_{\sigma}\right) \in T^{(\alpha)}$ has been defined. By the definition of the $T^{(\alpha+1)}$, we can have $\left(s_{0}, t_{0}\right),\left(s_{1}, t_{1}\right) \in T^{(\alpha)}$ such that both extend $\left(s_{\sigma}, t_{\sigma}\right)$ and $s_{0} \perp s_{1}$ and $\left(N_{s_{0}} \cap X\right) \times\left(N_{s_{1}} \cap X\right) \subseteq K_{0}$. Then let $s_{\widehat{\sigma\langle 0\rangle}}=s_{0}, s_{\widehat{\sigma\langle 1\rangle}}=s_{1}$. Similarly define the $t^{\prime} s$.

Finally, let $F=\left\{s_{\sigma} \mid \sigma \in 2^{<\omega}\right\}$. By the definition, we have that $F$ is a perfect tree, i.e., for each $s \in F$ there are $s_{0} \perp s_{1}$ such that $s_{0} \leq s$ and $s_{1} \leq s$. Furthermore, $F \in L[a],[F] \subseteq p\left[T^{(\alpha)}\right]$, and $[F]^{2} \subseteq K_{0}$.

This finishes the proof.

Corollary 2.1 (Mansfield and Solovay). Let $X$ be a $\Sigma_{2}^{1}(a)$ subset of the reals. Then either there is a perfect subset $P \subseteq X$ or $X \subseteq L[a]$.

Proof. Take $K_{1}=\varnothing$, i.e., the trivial partition. If there is a perfect 0 -homogeneous set, we are done. If not, then for each $y \in X$ there is a tree $F \in L[a]$ on $\omega$ such that $y \in[F]$ and $[[F] \cap X]^{2} \subseteq K_{1}$. So in this case, let $y \in X$ and let $F \in L[a]$ be a tree witnessing the truth of the conclusion. Then $[F] \cap X=\{y\}$. By absoluteness, there is at least one element in $[F] \cap X \cap L[a]$. So $y \in L[a]$.

Corollary 2.2. The following are equivalent: (1) $\forall a \subseteq \omega \aleph_{1}^{L[a]}<\aleph_{1}$.

(2) $\operatorname{OCA}\left(\underline{\Sigma}_{2}^{1}\right)$.

(3) $\operatorname{OCA}\left(\underline{\boldsymbol{\Pi}}_{1}^{1}\right)$.

Proof. Assume that $X$ is a $\Sigma_{2}^{1}(b)$ set and $[X]^{2}=K_{0} \cup K_{1}$ is a partition with $K_{0}$ relatively open. Let $c$ be a code for the open partition. Let $a=c * b$. Consider the model $L[a]$. By the theorem above, $(1) \Rightarrow(2)$. Since $\operatorname{OCA}\left(\underline{\boldsymbol{I}}_{1}^{1}\right)$ implies that every uncountable $\underline{I}_{1}^{1}$ set contains a perfect subset, (1) follows from (3).

Remark. Assume that every $\underline{\Pi}_{1}^{1}$ set is determined (see the next section for the explanation). Also assume that there is no $0^{\dagger}$ (see Jech [10]). For each real $a \in \mathbb{R}$, let $K_{a}$ be the Dodd and Jensen core model [5]. Then all the results of this section remain true substituting $L[a]$ by $K_{a}, \underline{\Sigma}_{2}^{1}$ by $\underline{\Sigma}_{3}^{1}$, and $\underline{\Pi}_{1}^{1}$ by $\underline{\Pi}_{2}^{1}$ everywhere. One uses the Jensen $\underline{\Sigma}_{3}^{1}$ absoluteness [11] instead of Shoenfield $\Sigma_{2}^{1}$ absoluteness.

\section{A GAME FOR OPEN COLORING OF PAIRS OF REALS}

Various forms of determinacy can be thought as natural challenges to the well orderability of the reals. Such challenges provide us very different structural realms of the reals. In the past 30 years or so, determinacy has been proven very powerful in giving various regularity properties to subsets of the reals, which are usually assumed by the analytic sets, like being Lebesgue measurable, having the property of Baire, being Ramsey, etc. 
In this section, we show how determinacy gives partition properties of the plane.

First let us classify what we mean by determinacy.

Given a subset $X \subseteq \mathbb{R}=\omega^{\omega}$ of the reals, we consider a two person game $\mathscr{G}(X)$ associated to $X$ :

$$
\begin{array}{lllllll}
\text { I } & n_{0} & & n_{1} & & \ldots & x \\
\text { II } & & m_{0} & & m_{1} & \ldots & y
\end{array}
$$

Player I and II alternately pick natural numbers in countably many steps, producing two reals $x$ and $y$. Let $a=x * y$, i.e., $a(2 n)=x(n)$ and $a(2 n+1)=$ $y(n)$. Then the payoff of the game is that I wins if and only if $a \in X$.

We are interested in for which $X$ one of the players always wins. To be more precise, a strategy is a function $f: \omega^{<\omega} \rightarrow \omega$. A play $a \in \omega^{\omega}$ is a play according to the strategy $f$ for I if for each $n$ we have $f(a\lceil 2 n)=a(2 n)$. A strategy $f$ is a winning strategy for I if for each play $a$ according to $f$ for I we have I wins, i.e., $a \in X$. Similarly we define winning strategies for II. We say that $X \subseteq \mathbb{R}$ is determined if one of the players has a winning strategy in the game $\mathscr{G}(X)$.

By PD we mean the statement that every projective subset of the reals is determined. By AD we mean the statement that every subset of the reals is determined. We should point out that $\mathrm{AD}$ and $\mathrm{AC}$ are contradictory to each other, while by the recent work of Martin and Steel [13, 14], PD is consistent with $\mathrm{ZF}+\mathrm{AC}$, and by the work of Martin and Steel and Woodin [13, 14, 24], $\mathrm{AD}$ is consistent with $\mathrm{ZF}+\mathrm{DC}$, assuming in both cases that large cardinals are consistent.

Now let us define a type of game associated to open partitions.

Since $\operatorname{OCA}(X)$ implies $X$ is either countable or contains a perfect subset, it is not surprising that our game is a variant of the $M$. Davis game [4], and the proof is similar. (The relation of the two games will be clear later.)

Given $X \subseteq 2^{\omega}$ and an open partition $[X]^{2}=K_{0} \cup K_{1}$ with $K_{0}$ open in the relative topology, we consider the following game $\mathscr{G}\left(X, K_{0}, K_{1}\right)$ associated with this partition.

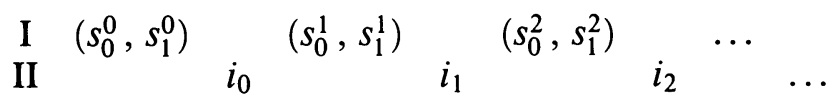

The rules of the play are the following:

(1) $i_{n}$ is either 0 or 1 .

(2) $s_{0}^{n}, s_{1}^{n}$ are nonempty finite sequences of 0 's and 1 's so that there is a $k$ so that $s_{0}^{n}(k) \neq s_{1}^{n}(k)$.

$$
(X \cap N \overbrace{s_{i_{0} s_{i_{1}}^{0} \cdots s_{0}^{n}}^{1}}) \times(X \cap N \overbrace{s_{i_{0}}^{0} \cdots s_{1}^{n}}) \subseteq K_{0} \quad \text { for } n \geq 0 .
$$

The output of the play is a real $x$ defined by

$$
x=\overbrace{s_{i_{0}}^{0} s_{i_{1}}^{1} \cdots s_{i_{n}}^{n} \cdots} .
$$

We define that I wins the play if and only if I obeys all the rules of the play and $x \in X$. 
Lemma 3.1. I has a winning strategy if and only if $X$ contains a perfect set which is 0-homogeneous.

Proof. Let $\sigma$ be a winning strategy for I. Then $\sigma$ induces a one-to-one continuous function $f: 2^{\omega} \rightarrow X$ such that $f^{\prime \prime}\left[2^{\omega}\right]$ is 0 -homogeneous.

For the other direction, I simply plays along a perfect tree which witnesses the 0 -homogeneity of a perfect set.

Lemma 3.2. II has a winning strategy if and only if $X$ is a countable union of 1-homogeneous sets.

Proof. $(\Leftarrow)$. Let $X=\bigcup_{n<\omega} X_{n}$ be a countable union of 1-homogeneous sets. We define a winning strategy $\tau$ for II as follows.

Assume $\left(s_{0}^{0}, s_{1}^{0}\right)$ is a first legal move of $I$. Then either $N_{s_{0}^{0}} \cap X_{0}=\varnothing$ or $N_{s_{1}^{0}} \cap X_{0}=\varnothing$. Then $i_{0}=0$ if the first equation holds and $i_{0}=1$ otherwise.

Inductively, assume $i_{m}$ are defined for $m \leq n$ and if $s=s_{i_{0}}^{0} s_{i_{1}}^{1} \cdots s_{i_{n}}^{n}$, then for each $m \leq n$ we have $N_{s} \cap X_{m}=\varnothing$. Let $\left(s_{0}^{n+1}, s_{1}^{n+1}\right)$ be a next legal move of I. Then either $N_{\widehat{s s_{0}^{n+1}}} \cap X_{n+1}=\varnothing$ or $N_{\widehat{s s_{1}^{n+1}}} \cap X_{n+1}=\varnothing$. So let $i_{n+1}=0$ if the first equation holds and let $i_{n+1}=1$ otherwise.

This defines a strategy $\tau$ for II. It wins. Assume that I obeys all the rules in a play $x$ in which II plays according to $\tau$. Then $x \notin X$. Otherwise, let $n$ be the least such that $x \in X_{n}$. Let $s=s_{i_{0}}^{0} s_{i_{1}}^{1} \cdots s_{i_{n}}^{n}$. Then $s \subseteq x$ and $N_{s} \cap X_{n}=\varnothing$. Contradiction.

$\Rightarrow$ Assume that II has a winning strategy $\tau$. We are going to find a decomposition of $X$ into countable union of 1-homogeneous sets.

First of all, for $s \in 2^{<\omega}$, let

$$
B_{s}=\left\{s \in X \mid s \subseteq x \& \forall y \in N_{s} \cap X[\{x, y\}]^{2} \subseteq K_{1}\right\} .
$$

Then $\left[B_{s}\right]^{2} \subseteq K_{1}$.

Let $X_{0}=\bigcup\left\{B_{s} \mid s \in 2^{<\omega}\right\}$, and $A=X-X_{0}$. Then

$$
\forall x \in A \forall n<\omega \exists y \in N_{x \mid n} \cap X\{x, y\} \in K_{0} .
$$

Main Claim. $A$ is a countable union of 1 -homogeneous sets.

The main claim gives the lemma. We now proceed to prove the main claim.

We say that $p$ is a correct position if $p$ is a lawful partial play of finite length in which II played following the winning strategy $\tau$ and II made the last move of the partial play, i.e., $p$ is the following partial play:

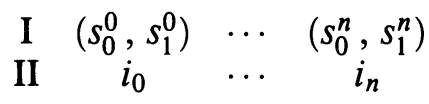

with all the responses of II are given by the winning strategy $\tau$ and no one breaks the rules.

For a correct position $p$, we define the output $s(p)$ of the partial play by $s(p)=s_{i_{0}}^{0} \cdots s_{i_{n}}^{n}$, where $n$ is the number of steps played in $p$. By convention, the empty sequence is a correct position and the output is the empty sequence.

We say that I gives up at a correct position $p$ if I cannot make a rext move without violating the rules.

Fact 1. If I gives up at a correct position $p$, then $N_{s(p)} \cap X$ is 1-homogeneous. 
Otherwise, let $x, y \in N_{s(p)} \cap X$ be such that $\{x, y\} \in K_{0} . K_{0}$ being open, there are two incompatible sequences $s, t$ in $2^{<\omega}$ such that $\overbrace{s(p) s} \subseteq x$, $\overbrace{s(p) t} \subseteq y$ and

$$
(\overbrace{s(p) s} \cap X) \times(\overbrace{s(p) t} \cap X) \subseteq K_{0} .
$$

Then I could play this pair without disobeying the rules.

Fact 2. Assume that $p$ is a correct position and $s(p) \subseteq x$. If there is no $(s, t)$ such that $\left(N_{s(p) s} \cap X\right) \times\left(N_{s(p) t} \cap X\right) \subseteq K_{0}$ and either $s(p)^{\wedge} s \subseteq x$ or $s(p)^{\wedge} t \subseteq x$, then $\forall y \in N_{s(p)} \cap X[\{x, y\}]^{2} \subseteq K_{1}$.

This again follows from the openness of $K_{0}$.

Fact 3. For $x \in 2^{\omega}$, assume that for each correct position $p, s(p) \subseteq x$ implies there is $(s, t) \in 2^{<\omega} \times 2^{<\omega}$ such that

$$
\left(N_{s(p) s} \cap X\right) \times\left(N_{s(p) t} \cap X\right) \subseteq K_{0}
$$

and

$$
s(p,(s, t), \tau(p,(s, t))) \subseteq x
$$

then $x \notin X$.

One simply builds by induction a play following $\tau$ with output $x$. Because $\tau$ wins the game, $x \notin X$.

We are now ready to decompose $A$ into a countable union of 1-homogeneous sets.

For a correct position $p$, we define that for $x \in A, x \in F_{p}$ if and only if $s(p) \subseteq x$ and for each next lawful move $(s, t)$ of $\mathrm{I}, s(p,(s, t), \tau(p,(s, t))) \nsubseteq$ $x$.

By the Fact 3, we have that $A=\bigcup\left\{F_{p} \mid p\right.$ is a correct position $\}$.

Claim. Each $F_{p}$ is a 1-homogeneous set.

Proof. If not, let $p$ be a correct position such that $F_{p}$ is not 1-homogeneous. Let $x, y$ be in $F_{p}$ such that $\{x, y\} \in K_{0}$.

Let $n$ be such that $x|n \neq y| n$ and $\left(N_{x \mid n} \cap X\right) \times\left(N_{y \mid n}\right) \subseteq K_{0}$. Then $s(p) \subseteq$ $x\lceil n$ and $s(p) \subseteq y\lceil n$. Let $(s, t)$ be such that $s(p) s=x\lceil n$ and $s(p) t=y \mid$ $n$. Then $(s, t)$ is a next lawful move of $\mathrm{I}$. But $s(p,(s, t), \tau(p,(s, t)))$ is then either an initial part of $x$ or an initial part of $y$.

This finishes the proof of the lemma.

We now consider a variant game $\mathscr{G}^{*}\left(X, K_{0}, K_{1}\right)$, which is equivalent to the above game.

As above, given $X \subseteq 2^{\omega}$ and an open partition $[X]^{2}=K_{0} \cup K_{1}$ with $K_{0}$ open in the relative topology, we consider the following game $\mathscr{G}^{*}\left(X, K_{0}, K_{1}\right)$ associated with this partition.

$$
\begin{array}{ccccc}
\text { I } & \left(s_{0}^{0}, s_{1}^{0}\right) & \left(s_{0}^{1}, s_{1}^{1}\right) & \left(s_{0}^{2}, s_{1}^{2}\right) & \ldots \\
\text { II } & \left(i_{0}, j_{0}\right) & \left(i_{1}, j_{1}\right) & \left(i_{2}, j_{2}\right) & \ldots
\end{array}
$$

The rules of the play are the following:

(1) $i_{n}, j_{n}$ are either 0 or 1 . 
(2) $s_{0}^{n}, s_{1}^{n}$ are nonempty finite sequences of 0 's and 1 's such that there is a $k$ such that $s_{0}^{n}(k) \neq s_{1}^{n}(k)$.

$$
(X \cap N \overbrace{s_{i_{0} j_{0} s_{i_{1}}^{1} \cdots s_{0}^{n}}^{n}}) \times(X \cap N \overbrace{s_{i_{0} j_{0} \cdots s_{1}^{n}}^{0}}) \subseteq K_{0} \text { for } n \geq 0
$$

(it is assumed here none of the intersections is empty). The output of the play is a real $x$ defined by

$$
x=\overbrace{s_{i_{0}}^{0} j_{0} s_{i_{1}}^{1} j_{1} \cdots s_{i_{n}}^{n} j_{n} \cdots} .
$$

We define that I wins the game if and only if I obeys all the rules of the game and $x \in X$.

Proposition. I (II) has a winning strategy in $\mathscr{G}\left(X, K_{0}, K_{1}\right)$ if and only if I (II) has a winning strategy in $\mathscr{G}^{*}\left(X, K_{0}, K_{1}\right)$.

It is easy to see that $I$ has a winning strategy in either game if and only if there is a perfect 0 -homogeneous set. So we need only show the equivalence of II winning the games.

Lemma 3.3. If II has a winning strategy in $\mathscr{G}\left(X, K_{0}, K_{1}\right)$, then II has a winning strategy in $\mathscr{G}^{*}\left(X, K_{0}, K_{1}\right)$.

Proof. Let $\tau$ be a winning strategy in the first game. We define a winning strategy $\tau^{*}$ for the game $\mathscr{G}^{*}\left(X, K_{0}, K_{1}\right)$ as follows.

$\tau^{*}$ will simulate the $\tau$ in the following pattern.

$\tau^{*}\left(\left(s_{0}^{0}, s_{1}^{0}\right)\right)=\left(\tau\left(\left(s_{0}^{0}, s_{1}^{0}\right)\right), 0\right)$.

If $\left(s_{0}^{1}, s_{1}^{1}\right)$ is a legal move of $\mathrm{I}$ in $\mathscr{G}^{*}\left(X, K_{0}, K_{1}\right)$, then $\left(\widehat{0 s_{0}^{1}}, \widehat{0 s_{1}^{1}}\right)$ is a legal move of $\mathrm{I}$ in the game $\mathscr{G}\left(X, K_{0}, K_{1}\right)$. So $\tau$ will give an $i_{1}$. Then $\tau^{*}$ plays $\left(i_{1}, 0\right)$ as the next move.

Continue in this way. Then the two plays produce the same real $x$, say. Unless I breaks the rules in some stage, because $\tau$ wins the game, $x \notin X$. Hence $\tau^{*}$ is a winning strategy.

Then the following lemma will finish the proof of the proposition.

Lemma 3.4. If II has a winning strategy in the star game, then $X$ is a countable union of 1-homogeneous sets.

This lemma is proved using the same arguments as in the proof of the Lemma 3.2. Let $\tau$ be a winning strategy for II in the star game. As in the proof of Lemma 3.2, define the correct partial play $p, s(p), F_{p}$ and $A$ in the same way. Now the following claim implies the lemma.

Claim. Each $F_{p}$ is a countable union of 1-homogeneous sets.

Proof. We will be using Facts 1 and 2 (as in the proof of Lemma 3.2) implicitly in the following arguments.

Case 1. $\left[F_{p}\right]^{2} \subseteq K_{1}$.

In this case, we are done.

Case 2. There are distinct $x, y$ in $F_{p}$ such that $\{x, y\} \in K_{0}$.

This is the case we have to make a decomposition.

We define

$$
D_{p}=\left\{(s, t) \mid \exists k s(k) \neq t(k),\left(N_{s(p) s} \cap X\right) \times\left(N_{s(p) t} \cap X\right) \subseteq K_{0}\right\} .
$$


Subclaim. For $(s, t) \in D_{p}$, we have either $N_{s(p) s} \cap F_{p}$ or $N_{s(p) t} \cap F_{p}$ has cardinality at most one.

Let us assume that both of the intersections are not empty. We show that we can uniquely determine one of the intersections.

Let $m_{0}=\operatorname{lh}(s(p) s)$ and $n_{0}=\operatorname{lh}(s(p) t)$. We have that $x \uparrow m_{0}$ and $y \uparrow n_{0}$ are determined. We are going to determine one of them by stages.

Stage 0. Continue the partial play $p$ by playing $(s, t)$.

If the winning strategy $\tau$ gives $(0, j)$, then define $x\left(m_{0}\right)=1-j$, and let $s_{0}=s(1-j), t_{0}=t$. If the winning strategy $\tau$ gives $(1, j)$, then define $y\left(n_{0}\right)=1-j$, and let $s_{0}=s, t_{0}=t(1-j)$.

Stage 1. Continue the partial play $p$ by playing $\left(s_{0}, t_{0}\right)$.

Let $m_{1}$ be the least such that $x\left(m_{1}\right)$ needs to be determined. Let $n_{1}$ be the least such that $y\left(n_{1}\right)$ needs to be determined. If $\tau$ gives $(0, j)$, then define $x\left(m_{1}\right)=1-j$, and let $s_{1}=s_{0}(1-j), t_{1}=t_{0}$. If $\tau$ gives $(1, j)$, then define $y\left(n_{1}\right)=1-j$, and let $s_{1}=s_{0}, t_{1}=t_{0}(1-j)$.

Stage $k+1$. Continue the partial play $p$ by playing $\left(s_{k}, t_{k}\right)$.

Let $m_{k+1}$ be the least such that $x\left(m_{k+1}\right)$ needs to be determined. Let $n_{k+1}$ be the least such that $y\left(n_{k+1}\right)$ needs to be determined. If $\tau$ gives $(0, j)$, then define $x\left(m_{k+1}\right)=1-j$, and let $s_{k+1}=s_{k}(1-j), t_{k+1}=t_{k}$. If $\tau$ gives $(1, j)$, then define $y\left(n_{k+1}\right)=1-j$, and let $s_{k+1}=s_{k}, t_{k+1}=t_{k}(1-j)$.

Notice that here we have used implicitly the Facts 1 and 2.

If the $\tau$ gives alternatively 0 's and 1 's infinitely often for the first coordinate, then we conclude that both intersections are singleton. If the $\tau$ gives eventually some constant for the first coordinate, then one of them is singleton.

For $(s, t) \in D_{p}$, we define that $E_{p,(s, t)}$ is one of the intersections whose cardinality is at most one, and $C_{p,(s, t)}$ is the other one if it also has cardinality at most one, otherwise, $C_{p,(s, t)}$ is the empty set. Let

$$
H_{p}=\bigcup\left\{E_{p,(s, t)}, C_{p,(s, t)} \mid(s, t) \in D_{p}\right\} .
$$

Finally, let $F_{p}^{*}=F_{p}-H_{p}$.

Our last claim is that $\left[F_{p}^{*}\right]^{2} \subseteq K_{1}$. To see this, let $x \neq y$ be in $F_{p}^{*}$ such that $\{x, y\} \in K_{0}$. Let $n$ be large enough so that $x \uparrow n \neq y \uparrow n$ and both extend $s(p)$ and $\left(N_{x \mid n} \cap X\right) \times\left(N_{y \mid n} \cap X\right) \subseteq K_{0}$. Then we have $(s, t) \in D_{p}$, where $s(p) s=x \uparrow n, s(p) t=y\left\lceil n\right.$. So either $x \in H_{p}$ or $y \in H_{p}$.

Therefore, the claim is proved.

Therefore, by Lemmas 3.2, 3.3, and 3.4, II has a winning strategy in $\mathscr{G}\left(X, K_{0}, K_{1}\right)$ if and only if $X$ is a countable union of 1 -homogeneous sets if and only if II has a winning strategy in $\mathscr{G}^{*}\left(X, K_{0}, K_{1}\right)$.

This finishes the proof of the proposition.

Remark. Originally we considered only the star game. After a seminar talk in MSRI, D. A. Martin suggested to consider the first game above by pointing out that it is easier for II to win the star game thus one should be able to get a more direct decomposition assuming that II has a winning strategy in the first game. Namely, there is no need of decomposition of the $F_{p}$ 's.

Remark. Notice that this game gives an alternative proof of $\operatorname{OCA}\left(\underline{\Sigma}_{1}^{1}\right)$. Since we can take $X=2^{\omega}$, the game is open for II, i.e., if II wins then II wins 
in finitely many steps. Hence it is determined by Gale and Stewart [26]. So $\operatorname{OCA}\left(2^{\omega}\right)$ holds. It follows that $\operatorname{OCA}(\mathbb{R})$, and therefore, $\operatorname{OCA}\left(\Sigma_{1}^{1}\right)$.

Remark. In conversations with the author, L. Harrington and $\mathrm{H}$. Woodin asked respectively how effective the decomposition of $\operatorname{OCA}\left(\Sigma_{1}^{1}\right)$ can be, in particular when the underlying set is Borel. The following remarks give an answer.

Applying Martin's Borel determinacy theorem [30] to the first game above, if $X$ is Borel (analytic) and the open partition $[X]^{2}=K_{0} \cup K_{1}$ has no perfect 0 -homogeneous subset, then $X$ is a countable union of 1 -homogeneous subsets $X=\bigcup_{n<\omega} X_{n}$ so that each $X_{n}$ is Borel (analytic) uniformly in $n$, i.e., there are two formulae $\varphi_{0}(x, n), \varphi_{1}(x, n)$ which finitely many real parameters such that $\varphi_{0}$ is $\underline{\Sigma}_{1}^{1}$ and $\varphi_{1}$ is $\underline{\Pi}_{1}^{1}$ and for each $n$ for each $x \in \mathbb{R}$ we have

$$
x \in X_{n} \Leftrightarrow \varphi_{0}(x, n) \Leftrightarrow \varphi_{1}(x, n) .
$$

(In case of analytic sets, only the $\underline{\Sigma}_{1}^{1}$ formula is required.)

This is because when $X \subseteq 2^{\omega}$ is Borel and dense in $2^{\omega}$, each $B_{s}$ defined in the proof of Lemma 3.2 is Borel uniformly in $s \in 2^{<\omega}$, and $F_{p}$ is Borel uniformly in $p$, while such $p$ form a subset of $\left(2^{<\omega} \times 2^{<\omega} \times 2\right)^{<\omega}$ which is recursive in some real parameters. If $X$ is not dense in $2^{\omega}$, by adding two reals coding the isolated points and a homeomorphism, we can reduce to the case dense in the Cantor space.

The reason for analytic sets is that every analytic set is a continuous image of the Baire space and every continuous function can be coded as a real in an effective way and every open partition on the analytic set induces canonically an open partition on the Baire space by the continuous function.

Finally, as applications of the above games, we conclude the following to end this section.

Theorem 3.1. If all $\underline{I}_{n}^{1}$ sets are determined, then $\operatorname{OCA}\left(\underline{\Sigma}_{n+1}^{1}\right)$. In particular, if PD holds, then OCA (Projective Set).

Theorem 3.2. If $A D^{L(\mathbb{R})}$ holds, then every subset $X$ of the reals which is in $L(\mathbb{R})$ satisfies $\mathrm{OCA}(X)$.

Theorem 3.3. $Z F+D C+A D \vdash \operatorname{OCA}(P(\mathbb{R}))$, where $P(\mathbb{R})$ is the family of all subsets of the reals.

Remark. It follows from the works of Martin, Steel, and Woodin that if there are sufficiently large cardinals then $\operatorname{OCA}(\Gamma)$ for $\Gamma$ the family of projective sets, or $\Gamma=L(\mathbb{R}) \cap P(\mathbb{R})$.

\section{OCA IN SolovaY's MODEL}

In this section we show first that in Solovay's model [27] OCA holds. The argument is essentially the Solovay's argument showing that in Solovay's model every uncountable set of reals has a perfect subset. I thank Todorcevic for convincing me that Solovay's argument should work. My contribution is merely to supply a proof of a key fact which makes the argument work. Then we present a forcing proof of $\operatorname{OCA}\left(\Sigma_{1}^{1}\right)$ due to Todorcevic.

First let us briefly review the Solovay's model which we now turn into.

Let $\kappa$ be a (strongly) inaccessible cardinal. Let $\operatorname{Col}(\omega,<\kappa)$ be the Levy collapsing to make $\kappa$ to be $\omega_{1}$. Each condition $p$ is a finite function with 
$\operatorname{dom}(p) \subseteq \kappa \times \omega$ and $p(\alpha, n)<\alpha$ whenever $(\alpha, n) \in \operatorname{dom}(p)$. The order is by extension.

Let $G$ be a generic. In $V[G]$, define the Solovay model to be the class of all sets hereditarily ordinal definable over the class of all infinite sequences of ordinals. We show that in this model OCA holds.

We will use the following basic properties of this model which are by now standard (see $[27,10])$.

Lemma 4.1. Let $X$ be countable sequence of ordinals in $V[G]$.

(1) There exists a post $\mathbb{P}$ in $V$ of size smaller than $\kappa$ such that $X \in V[G \cap \mathbb{P}]$.

(2) There exists a $V[X]$-generic filter $H$ for $\operatorname{Col}(\omega,<\kappa)$ such that $V[X][H]$ $=V[G]$.

Lemma 4.2. Let $A \in V[G]$ be a set of reals that is definable from a sequence $s$ of ordinals. Then there is a formula $\varphi$ such that in $V[G]$ for all reals $x \in \mathbb{R}$,

$$
x \in A \Leftrightarrow V[s][x] \vDash \varphi(s, x) .
$$

We now work in $V[G]$.

Let $A$ be a set of reals which is definable from a countable sequence of ordinals. Let $[A]^{2}=K_{0} \cup K_{1}$ be a partition such that $K_{0}$ is relatively open. Assume that $A$ is not a countable union of 1-homogeneous sets. By the lemmas above, we may assume without loss of generality that the partition is in the ground model and for some formula $\varphi$,

$$
x \in A \Leftrightarrow V[x] \vDash \varphi(x) .
$$

Since $A$ is not a countable union of 1-homogeneous sets, there is an $x \in$ $A$ such that $x$ is not a branch of any tree $T$ in $V$ on $\omega$ satisfying that $[[T]]^{2} \cap K_{0}=\varnothing$. Fix such an $x$. Let $\mathbb{P}$ be a poset of size smaller than $\kappa$ such that $x$ is $\mathbb{P}$-generic over $V$. Let $\dot{x}$ be a $\mathbb{P}$-name for $x$. Let $p \in G \cap \mathbb{P}$ be a condition such that $p \Vdash$ " $\dot{x}$ is a real and for every tree $T \in V$ on $\omega$ if $[[T]]^{2} \cap K_{0}=\varnothing$ then $\dot{x} \notin[T]$, and $V[\dot{x}] \vDash \varphi(\dot{x})$."

The following is the key fact allowing us to construct a perfect 0 -homogeneous subset of $A$.

Fact. For every $s \in \omega^{<\omega}$, for every $q \leq p$, if $q \Vdash s \subseteq \dot{x}$, then there are incompatible $s_{0}, s_{1} \in \omega^{<\omega}$, extending $s$, and $q_{0}, q_{1} \in \overline{\mathbb{P}}$, stronger than $q$, such that

$$
q_{0} \Vdash s_{0} \subseteq \dot{x}, \quad q_{1} \Vdash s_{1} \subseteq \dot{x}, \bigwedge[A]^{2} \cap\left(N_{s_{0}} \times N_{s_{1}}\right) \subseteq K_{0} .
$$
by

Assume otherwise. Let $s$ and $q$ be counterexamples. Define a tree $T$ on $\omega$

$$
t \in T \Leftrightarrow s \subseteq t \wedge \exists q^{\prime} \leq q\left(q^{\prime} \Vdash t \subseteq \dot{x}\right) .
$$

Since $q \Vdash s \subseteq \dot{x} \wedge \dot{x} \notin V, T$ is a perfect tree and $T \in V$. Since $x \in$ $A \cap[T], A \cap[T]$ cannot be 1-homogeneous. Let $a \neq b$ be in $A \cap[T]$ such that $\{a, b\} \in K_{0}$. Pick $n<\omega$ sufficiently large so that $a\lceil n \neq b \uparrow n$ and

$$
[A]^{2} \cap\left(N_{a \mid n} \times N_{b \mid n}\right) \subseteq K_{0} .
$$

Since $a$ and $b$ are in $[T] \cap A$, there are two incompatible $q_{0}$ and $q_{1}$, stronger than $q$, such that $q_{0} \Vdash a \uparrow n \subseteq \dot{x}$ and $q_{1} \Vdash b \uparrow n \subseteq \dot{x}$. This contradicts the fact that $(s, q)$ is a counterexample. 
Now we are ready to construct our 0 -homogeneous set.

Let $\left\langle D_{n} \mid n<\omega\right\rangle$ be an enumeration of dense subsets of $\mathbb{P}$ which are in $V$.

Let $p_{\varnothing} \leq p$ be in $D_{0}$ to decide $\dot{x}(0)$. Let $t_{\varnothing}$ be such that $p_{\varnothing} \Vdash t_{\varnothing}=\dot{x}\lceil 1$.

Given $p_{s}$ and $t_{s}$ for $s \in 2^{<\omega}$ such that $p_{s} \Vdash t_{s} \subseteq \dot{x}$. By the fact above, there are two conditions $p_{s\langle 0\rangle}$ and $p_{s\langle 1\rangle}$ in $D_{\mathrm{lh}(s)+1}$ and two incompatible $s_{0}$ and $s_{1}$ in $\omega^{<\omega}$ such that

(1) $t_{s} \subseteq s_{0}, t_{s} \subseteq s_{1}$,

(2) $p_{s\langle 0\rangle} \Vdash s_{0} \subseteq \dot{x} \wedge p_{s\langle 1\rangle} \Vdash s_{1} \subseteq \dot{x}$, and

(3) $[A]^{2} \cap\left(N_{s_{0}} \times N_{s_{1}}\right) \subseteq K_{0}$.

Let $t_{s\langle 0\rangle}=s_{0}$ and $t_{s\langle 1\rangle}=s_{1}$. Then $T=\left\{t_{s} \mid s \in 2^{<\omega}\right\}$ is a perfect tree.

For $a \in 2^{\omega}$, let $x_{a}=\bigcup_{n<\omega} t_{a \mid n}$ and

$$
g_{a}=\left\{q \in \mathbb{P} \mid \exists n \in \omega p_{a \mid n} \leq q\right\} .
$$

Then $g_{a}$ is a $\mathbb{P}$-generic over $V$ and $\dot{x} / g_{a}=x_{a}$. So each $x_{a} \in A$ and if $a \neq b$ then $\left\{x_{a}, x_{b}\right\} \in K_{0}$. Hence $[[T]]^{2} \subseteq K_{0}$.

We have therefore proved the following theorem.

Theorem 4.1. Assume that there exists an inaccessible cardinal.

(1) There is a model of $Z F+D C$ in which OCA holds.

(2) There is a model of ZFC in which OCA (projective) holds, in fact, if $X \in$ $(P(\mathbb{R}) \cap L(\mathbb{R}))$ then $\operatorname{OCA}(X)$ holds.

Remark. By Corollary 2.2, it is necessary to start with an inaccessible cardinal.

We now give the forcing proof of $\operatorname{OCA}\left({\underset{\Sigma}{1}}_{1}^{1}\right)$ due to Todorcevic.

As mentioned in the introduction, it suffices to show $\operatorname{OCA}(\mathbb{R})$.

Let $V$ be the universe of set theory. Let $K_{0} \subseteq[\mathbb{R}]^{2}$ be an open set. We assume that $\mathbb{R}$ is not a countable union of 1 -homogeneous sets. The goal is to show that there is a perfect 0 -homogeneous set.

Let $\mathbb{P}_{0}$ be the Levy collapsing $\operatorname{Col}\left(\omega_{1}, 2^{\aleph_{0}}\right)$ via countable partial functions to make the continuum hypothesis hold. Let $G_{0}$ be $\mathbb{P}_{0}$-generic over $V$. Let $V_{1}=V\left[G_{0}\right]$ be the generic extension. Then in $V_{1}, \mathbb{R}$ is still not a countable union of 1-homogeneous sets, since there is no new countable sequence of closed sets in $V_{1}$.

By the work of $[20$, p. 71$]$, in $V_{1}$, there is an uncountable subset $Y \subseteq \mathbb{R}$ such that $\mathbb{P}_{1}=\left\{F \in[Y]^{<\omega} \mid[F]^{2} \subseteq K_{0}\right\}$, ordered by extension, is a ccc poset (i.e., having the countable chain condition). Let $G_{1}$ be $\mathbb{P}_{1}$-generic over $V_{1}$. Then in the generic extension $V_{2}=V_{1}\left[G_{1}\right]$, there is an uncountable 0 -homogeneous set.

Let $\mathbb{P}_{2}$ be a ccc forcing in $V_{2}$ to force $M A_{\omega_{1}}$ as defined in [29]. Here $M A_{\omega_{1}}$ is the statement that for every ccc forcing $P$ for every family of dense subsets of $P$ of size at most $\aleph_{1}$ there is a filter on $P$ meeting every dense subset in the family.

Let $G_{2}$ be $\mathbb{P}_{2}$-generic over $V_{2}$. Let $V_{3}=V_{2}\left[G_{2}\right]$ be the generic extension. Then in $V_{3}, M A_{\omega_{1}}$ holds and there is an uncountable 0-homogeneous set.

Claim. In $V_{3}$, there is a perfect 0 -homogeneous set.

The claim gives the theorem. Since the statement "there is a perfect set $P \subseteq \mathbb{R}$ such that $[P]^{2} \subseteq K_{0}$ " is $\Sigma_{2}^{1}\left(K_{0}\right)$. By Shoenfield absoluteness [18], it must be true in $V$.

So we need only show this claim. It follows from the following lemma, since every uncountable $G_{\delta}$-set contains a perfect subset. 
Lemma 4.3. Let $X \subseteq \mathbb{R}=\omega^{\omega}$ and $[X]^{2}=K_{0} \cup K_{1}$ be an open partition with $K_{0}$ relatively open. Assume that $A \subseteq X$ is an uncountable 0-homogeneous set, and $M A_{\omega_{1}}$ holds. Then there is $G_{\delta}$-set $B \supseteq A$ such that $[B \cap X]^{2} \subseteq K_{0}$.

Proof. Assume that $|A|=\aleph_{1}$. Consider the following poset $\mathbb{P}$.

A condition $p \in \mathbb{P}$ is a pair $p=\left\langle e_{p}, A_{p}\right\rangle$ satisfying the following:

(1) $A_{p} \in[A]^{<\omega}$ and $e_{p} \in\left[\omega^{<\omega}\right]^{<\omega}$,

(2) for all $s, t \in e_{p}, s \neq t \Rightarrow \operatorname{lh}(s)=\operatorname{lh}(t)$ and $\left(N_{s} \cap X\right) \times\left(N_{t} \cap X\right) \subseteq K_{0}$,

(3) for each $x \in A_{p}$ there is an $s \in e_{p}$ so that $x \in N_{s}$ and for each $s \in e_{p}$ there is an $x \in A_{p}$ such that $x \in N_{s}$.

The order of $\mathbb{P}$ is defined as follows:

$$
p \leq q \Leftrightarrow A_{q} \subseteq A_{p} \wedge \forall s \in e_{q} \exists t \in e_{p} \exists m(t \mid m=s) .
$$

It is easy to see that $\mathbb{P}$ is ccc.

For each $a \in A$ and $m<\omega$, let

$$
D_{a, m}=\left\{p \in \mathbb{P} \mid \exists s \in e_{p} \operatorname{lh}(s) \geq m \wedge a \in N_{s}\right\} .
$$

Then $D_{a, m}$ is dense in $\mathbb{P}$.

Applying $M A_{\omega_{1}}$, we get a filter $G$ meeting all of the $D_{a, m}$ 's. Let

$$
N=\left\{n \mid \exists p \in G \exists s \in e_{p}(n=\operatorname{lh}(s))\right\} .
$$

For each $n \in N$, let

$$
e_{n}=\bigcup\left\{e_{p} \mid p \in G \wedge \exists s \in e_{p}(n=\operatorname{lh}(s))\right\} .
$$

Finally, let

$$
B=\left\{a \in \omega^{\omega} \mid \forall m \exists n \in N\left(n \geq m \wedge \exists s \in e_{n}\left(a \in N_{s}\right\}\right)\right) .
$$

Then $A \subseteq B$, and $B$ is a $G_{\delta}$-set and $[B \cap X]^{2} \subseteq K_{0}$.

From the proof above, we have immediately the following corollary.

Corollary 4.1. If for every ccc forcing $\mathbb{P}$ in $V, \mathbb{P}$ forces that "every uncountable $\underline{\Pi}_{1}^{1}$ set contains a perfect subset," then in $V$, OCA $\left(\underline{\Sigma}_{2}^{1}\right)$ holds.

Given two models $\mathbb{M} \subseteq \mathbb{N}$ of set theory, we say that $\mathbb{N}$ is $\Sigma_{n}^{1}$ absolute with respect to $\mathbb{M}$ if $\mathbb{R} \cap \mathbb{M}$ is a $\Sigma_{n}$ elementary submodel of $\mathbb{R} \cap \mathbb{N}$. And $\mathbb{N}$ is absolute with respect to $\mathbb{M}$ if it is $\Sigma_{n}^{1}$ absolute for every $n$.

Then the above corollary says that if every ccc set forcing extension is $\Sigma_{4}^{1}$ absolute, then $\operatorname{OCA}\left(\Sigma_{2}^{1}\right)$ holds. If we allow non-ccc forcing, we would need only $\Sigma_{3}^{1}$ absoluteness. Namely, if every generic extension via a set forcing of size at most $\aleph_{1}$ is $\Sigma_{3}^{1}$ absolute, then $\operatorname{OCA}\left({\underset{\Sigma}{2}}_{2}^{1}\right)$ holds. This is because, given $a \in \mathbb{R}$, the statement " $\mathbb{R} \cap L[a]$ is countable" is a $\Sigma_{3}^{1}(a)$ statement, which is true in the generic extension via $\operatorname{Col}\left(\omega, \omega_{1}\right)$.

Remark. If every generic extension with forcing of size at most $2^{\aleph_{1}}$ is $\Sigma_{n+3}^{1}$ absolute with respect to $V$, then in $V$, OCA $\left(\Sigma_{n+1}^{1}\right)$ holds.

Namely, given a $\underline{I}_{n}^{1}$ set $X$ and $[X]^{2}=K_{0} \cup K_{1}$ with $K_{0}$ open, assume that $X$ is not a countable union of 1 -homogeneous sets. By the above forcing arguments and by $\Sigma_{n+3}^{1}$ absoluteness, one gets an uncountable $\underline{\sim}_{n}^{1}$ 0-homogeneous 
subset. To get that every uncountable $\underline{\Pi}_{n}^{1}$ subset contains a perfect subset, $\Sigma_{n+2}^{1}$ absoluteness is sufficient.

\section{SOME APPLICATIONS}

We have already seen some applications in the previous sections. Here we list some more.

To begin with, let us remark an amusing application. Let $\mathbb{R}$ be the real line. It follows from $\operatorname{OCA}(\mathbb{R})$ that every open set is a disjoint countable union of open intervals.

Filipczak in [7] showed that every continuous function from the reals to the reals is monotonic on a perfect set. We show there is much more to be said.

Lemma 5.1. Let $X \subseteq \mathbb{R}$. If $\operatorname{OCA}(X)$ holds, then for each continuous function $f: X \rightarrow \mathbb{R}$, either there is a perfect subset $P \subseteq X$ such that $f \uparrow P$ is strictly increasing, or $X=\bigcup_{n=1}^{\infty} X_{n}$ such that for each $n$, for each $x, y \in X_{n}, x \leq$ $y \Rightarrow f(y) \leq f(x)$, and each $X_{n}$ is relatively closed.

Proof. Let $f: X \rightarrow \mathbb{R}$ be continuous. Define a partition on $[X]^{2}$ by

$$
\begin{aligned}
& \{x, y\}_{<} \in K_{0} \Leftrightarrow f(x)<f(y), \\
& \{x, y\}_{<} \in K_{1} \Leftrightarrow f(y) \leq f(x) .
\end{aligned}
$$

The continuity of $f$ gives us the relative openness of $K_{0}$. Now apply $\operatorname{OCA}(X)$ to finish the proof.

Theorem 5.1. 1. If $A \subseteq \mathbb{R}$ is analytic, and $f: A \rightarrow \mathbb{R}$ is continuous, then either there is a perfect subset $P \subseteq X$ such that $f \uparrow P$ is either strictly increasing or strictly decreasing, or $f$ is a countable union of constant functions.

2. If $f: \mathbb{R} \rightarrow \mathbb{R}$ is a function whose graph is analytic, then either there is a perfect set $P$ such that $f \backslash P$ is either strictly increasing or strictly decreasing, or there is a dense $G_{\delta}$ set $A$ such that $f \mid A$ is a countable union of constant functions.

Proof. Let $A \subseteq \mathbb{R}$ be analytic. Let $f: A \rightarrow \mathbb{R}$ be continuous. If $A$ is countable, we are done. If there is a perfect subset $P \subseteq A$ such that $f \uparrow P$ is strictly increasing, we are done. Otherwise, by the lemma,

$$
A=\bigcup_{n=1}^{\infty} X_{n},
$$

with each $X_{n}$ is relatively closed and $f \uparrow X_{n}$ is monotonic and nonincreasing.

For each $n$, consider the partition $\left[X_{n}\right]^{2}=K_{0}^{n} \cup K_{1}^{n}$ induced by $f \uparrow X_{n}$, i.e.,

$$
\begin{aligned}
& \{x, y\}_{<} \in K_{0}^{n} \Leftrightarrow f(y)<f(x), \\
& \{x, y\}_{<} \in K_{1}^{n} \Leftrightarrow f(y)=f(x) .
\end{aligned}
$$

Since each $X_{n}$ is analytic, we have that either there is a perfect 0 -homogeneous subset, or $X_{n}$ is a countable union of 1-homogeneous sets. So if there is some $n$ such that there is a perfect 0 -homogeneous subset $P \subseteq X_{n}$, then we have that $f \uparrow P$ is strictly decreasing. If there is no such $n$, then each $X_{n}$ is a countable union of 1-homogeneous sets, i.e., $f \uparrow X_{n}$ is a countable union of constant functions for each $n$. Hence $f$ is a countable union of constant functions. 
This gives (1).

Now (2) follows from (1) by noticing that any such function is continuous on a dense $G_{\delta}$ subset. [Let $f$ be such a function. Let $\left\{I_{n} \mid n<\omega\right\}$ be an enumeration of all the rational open intervals of the real line. Then for each $n<\omega, f^{-1}\left[I_{n}\right]$ is an analytic subset of the real line. Hence they all have the Baire property. Let $B_{n}$ be open and $C_{n}$ be a countable union of closed nowhere dense sets such that $B_{n} \Delta f^{-1}\left[I_{n}\right] \subseteq C_{n}$ for each $n<\omega$. Let $A$ be the complement of $\bigcup C_{n}$. Then $A$ is a dense $G_{\delta}$ set and $f\lceil A$ is continuous.]

Remark. If $\underline{\Delta}_{2}^{1}$ be the family of sets which are $\underline{\Sigma}_{2}^{1}$ and whose complements are $\underline{\Sigma}_{2}^{1}$. If every $\underline{\Delta}_{2}^{1}$ subset of the reals has the property of Baire, then every coanalytic function $f: \mathbb{R} \rightarrow \mathbb{R}$ has the property stated in (2) above. Under PD, similar statement for projective functions from the reals to the reals holds.

Our next application is to consider the chain decomposition of closed partial orders on subsets of the reals. In [9], Harrington and Shelah proved the following theorem:

Let $\leq$ be a Borel partial order of the reals (i.e., $\leq \subseteq \mathbb{R} \times \mathbb{R}$ is a Borel subset of the plane which is a partial order of the reals). Then either there is a perfect set $P$ such that any two distinct elements of $P$ are $\leq$-incomparable, or $\mathbb{R}=\bigcup_{n=1}^{\infty} X_{n}$ with each $X_{n}$ is a $\leq$-chain.

In [21], Todorcevic gave some examples showing that a certain natural conjecture extending this result to a result that would give similar regularity property to all Borel ccc posets if false.

Here we point out the following immediate consequence of both the result of Harrington and Shelah and of $\operatorname{OCA}\left(\underline{\Sigma}_{1}^{1}\right)$.

If $X \subseteq \mathbb{R}$ is analytic, $\leq$ is a relatively closed partial order on $X$, then either there is a perfect set $P \subseteq X$ such that any two distinct elements of $P$ are $\leq$-incomparable, or $X$ is a countable union of $\leq$-chains.

This follows from the following fact.

Fact. If $\operatorname{OCA}(X)$ and $\leq$ is partial order on $X$ which is relatively closed. Then either there is a perfect subset whose elements are mutually $\leq$-incomparable or $X$ is a countable union of $\leq$-chains.

Let us mention a recent result of Todorcevic (see [21]) which is the dual statement of the above fact. That is, if $X \subseteq \mathbb{R}$ is analytic, $\leq$ is a partial order on $X$ which is relatively closed, then either there is a perfect $\leq$-chain, or $X$ is a countable union of subsets whose elements are mutually $\leq$-incomparable.

In the next applications, we assume $\mathrm{ZF}+\mathrm{DC}+\mathrm{AD}$.

First, we want to mention a well-known fact (e.g. [3]) which shows that the Sierpinski partition depends on essentially the axiom of choice.

Fact $(\mathrm{ZF}+\mathrm{DC}+\mathrm{AD})$. If $\left[2^{\omega}\right]^{2}=K_{0} \cup K_{1}$ is a partition, then there is a perfect homogeneous set.

Proof. Since AD holds, we can find two open sets $A, B$ such that $K_{0} \Delta A$ and $K_{1} \Delta B$ are meager. Now by Mycielski's theorem [15], we can find a perfect set $P \subseteq 2^{\omega}$ such that $[P]^{2} \subseteq\left(K_{0} \cap A\right) \cup\left(K_{1} \cap B\right)$. Then by $\operatorname{OCA}(P)$, either there is a perfect 0 -homogeneous subset of $P$, or $P$ is a countable union of closed 1-homogeneous sets, and hence there is a perfect 1-homogeneous subset.

Next we consider two statements, which are known in appropriate forms to be 
consistent with the axiom of choice [2, 20]. We point out here that they follow from $\mathrm{AD}$. Obviously, they are motivated by the corresponding consequences of OCA mentioned in $[20, \S 8]$.

Consider the partial order $(P(\omega), \subseteq) . X \subseteq P(\omega)$ is an antichain if for all $a, b \in X$ we have $a \nsubseteq b$ and $b \nsubseteq a . X \subseteq P(\omega)$ is a chain if for all $a, b \in X$ either $a \subseteq b$ or $b \subseteq a$. $X \subseteq P(\omega)$ is perfect if $X$ is perfect in the topology of $P(\omega)$ identified with $2^{\omega}$.

Fact $(\mathrm{ZF}+\mathrm{DC}+\mathrm{AD})$. Every subset of $(P(\omega), \subseteq)$ either has a perfect $\subseteq$ antichain or is a union of countably many $\subseteq$-chains, i.e., a Dilworth theorem holds for subposets of $P(\omega)$.

Proof. Given $X \subseteq p(\omega)$, define a partition $[X]^{2}=K_{0} \cup K_{1}$ by letting

$$
\{x, y\} \in K_{0} \Leftrightarrow x \nsubseteq y \wedge y \nsubseteq x .
$$

Then $K_{0}$ is relatively open.

Now apply $\operatorname{OCA}(X)$ to finish the proof.

Fact $(\mathrm{ZF}+\mathrm{DC}+\mathrm{AD})$. Every uncountable subalgebra $B$ of $P(\omega)$ has a perfect $\subseteq$-antichain.

Proof. Let $B$ be an uncountable subalgebra. Let $P \subseteq B$ be a perfect subset. Consider the partition $[P]^{2}=K_{0} \cup K_{1}$ with

$$
\{x, y\} \in K_{0} \Leftrightarrow x \nsubseteq y \wedge y \nsubseteq x .
$$

Then $K_{0}$ is open. So by $\operatorname{OCA}\left({\underset{\Sigma}{1}}_{1}^{1}\right)$, either there is a perfect antichain or there is a perfect chain. Assume there is a perfect chain. Let $Q$ be such a perfect chain.

Let $a$ be such that both of the following two sets are perfect.

$Q_{1}=\{b \in Q \mid b \subseteq a\}$.

$Q_{2}=\{b \in Q \mid a \subseteq b\}$.

Fix $f: Q_{1} \rightarrow Q_{2}$ some order isomorphism. Then $A=\left\{b \cup(-f(b)) \mid b \in Q_{1}\right\}$ is an uncountable Borel antichain. We are done.

Remark. In the above, we need only the consequence of AD that every uncountable subset of the reals contains a perfect subset.

\section{A REMARK ON A PARTITION THEOREM ON ERDÖS AND RADO}

Under the Axiom of Choice, Sierpiński's partition shows that there is an $f:\left[2^{\aleph_{0}}\right]^{2} \rightarrow 2$ such that there is no uncountable homogeneous set. In [6], Erdös and Rado showed that assuming the axiom of choice, if $f:\left[\left(2^{\aleph_{0}}\right)^{+}\right]^{2} \rightarrow \omega$, then there is an uncountable homogeneous set, i.e., in the notation of Erdös and Rado,

$$
\left(2^{\aleph_{0}}\right)^{+} \rightarrow\left(\omega_{1}\right)_{\omega}^{2}
$$

where $\lambda \rightarrow\left(\omega_{1}\right)_{\omega}^{2}$ if and only if for every $f:[\lambda]^{2} \rightarrow \omega$ there is an uncountable $H \subseteq \lambda$ such that $f \mid[H]^{2}$ is a constant.

In this remark, we show that a stronger form of Erdös-Rado theorem holds even in the context of lacking a well order of the reals.

Let us define that

$$
\boldsymbol{\Theta}=\sup \{\lambda \mid \exists \pi: \mathbb{R} \stackrel{\text { onto }}{\rightarrow} \lambda\} .
$$


Then in the theory of ZF+DC, $\Theta$ is a cardinal of cofinality $>\omega$. If $A C$ is assumed, then $\boldsymbol{\theta}=\left(2^{\aleph_{0}}\right)^{+}$. The stronger form of the Erdös-Rado theorem we have in mind is the following.

Theorem 6.1 $(\mathrm{ZF}+\mathrm{DC})$. If $\boldsymbol{\Theta}$ is regular, then $\boldsymbol{\Theta} \rightarrow\left(\aleph_{1}\right)_{\omega}^{2}$.

Corollary 6.1 (Erdös and Rado). If AC holds, then $\left(2^{\aleph_{0}}\right)^{+} \rightarrow\left(\aleph_{1}\right)_{\omega}^{2}$.

We now proceed to prove the theorem.

Let $f:[\Theta]^{2} \rightarrow \omega$ be given. We would like to find an uncountable homogeneous set for this $f$.

From $f$ we get naturally a sequence of functions from $\Theta$ to $\omega$. That is, for $\alpha<\boldsymbol{\Theta}$ let

$$
f_{\alpha}(\beta)= \begin{cases}f(\{\alpha, \beta\}) & \text { if } \alpha \neq \beta, \\ 0 & \text { if } \alpha=\beta .\end{cases}
$$

Claim. For each $\alpha<\boldsymbol{\theta}$ for each $x \in[\alpha]^{\aleph_{0}}$, there is a $\beta<\boldsymbol{\theta}$ such that $\beta>\alpha$ and

$$
\forall \gamma<\boldsymbol{\theta} \gamma>\beta \Rightarrow \exists \rho\left(\alpha \leq \rho<\beta \wedge f_{\rho}\left|x=f_{\gamma}\right| x\right) .
$$

Assume otherwise. Let $\alpha$ be the least counterexample. Let $x \in[\alpha]^{\aleph_{0}}$ be a witness to that. That is, we have

$$
\forall \beta<\boldsymbol{\theta} \beta>\alpha \Rightarrow \exists \gamma<\boldsymbol{\Theta}\left(\gamma>\beta \wedge \forall \rho\left(\alpha \leq \rho<\beta \wedge f_{\rho}\left|x \neq f_{\gamma}\right| x\right)\right) .
$$

We are going to define a one-to-one function from $\Theta$ to $\mathbb{R}$ which will be a contradiction.

Define two functions $h$ and $F$ by induction as follows:

$h(0)=$ the least $\gamma>\alpha+1$ such that $f_{\gamma}\left\lceil x \neq f_{\alpha} \mid x\right.$.

$F(0)=f_{h(0)}\lceil x$.

Let $\lambda<\Theta$. Inductively assume that $F \mid \lambda$ and $h \mid \lambda$ are defined and satisfying the following conditions:

(1) $F: \lambda \stackrel{1-1}{\longrightarrow} \omega^{x}$;

(2) $\forall i<\lambda F(i)=f_{h(i)}\lceil x$.

(3) $h$ is strictly increasing.

Let $\beta=\sup \{h(i) \mid i<\lambda\}$. Since $\boldsymbol{\theta}$ is regular, $\beta<\boldsymbol{\theta}$. So define $h(\lambda)$ to be the least $\gamma<\Theta$ such that $\gamma>\beta$ and

$$
\forall \rho\left(\alpha \leq \rho<\beta \wedge f_{\rho} \mid x \neq f_{\gamma}\lceil x) .\right.
$$

Then let $F(\lambda)=f_{h(\lambda)} \mid x$.

Since $x$ is countable, we have $\omega^{x} \cong \mathbb{R}$. Therefore, there is a one-to-one mapping from $\Theta$ to $\mathbb{R}$. But this is impossible. This proves the claim.

We would like to define a continuous increasing sequence $\left\langle\gamma_{\xi} \mid \xi<\omega_{1}\right\rangle$ of length $\omega_{1}$ from $\boldsymbol{\theta}$ satisfying the following properties:

Letting $\eta=\sup \left\{\gamma_{\xi} \mid \xi<\omega_{1}\right\}$, then for all $\xi<\omega_{1}$, if $x \in\left[\gamma_{\xi}\right]^{\aleph_{0}}$, then there is a $\rho$ such that

$$
\gamma_{\xi} \leq \rho<\gamma_{\xi+1} \wedge f_{\rho} \mid x=f_{\eta}\lceil x .
$$

First let us observe that if $\gamma<\boldsymbol{\Theta}$, if $g:[\gamma]^{\mathrm{N}_{0}} \rightarrow \boldsymbol{\Theta}$, then there is a $\lambda<\boldsymbol{\theta}$ such that for each $y \in[\gamma]^{\aleph_{0}} g(y)<\lambda$.

This is because under ZF + DC, if $\pi: \mathbb{R} \stackrel{\text { onto }}{\rightarrow} \gamma$, then $\pi^{*}: \mathbb{R}^{\omega} \stackrel{\text { onto }}{\rightarrow} \gamma^{\omega}$, where $\pi^{*}(s)(n)=\pi(s(n))$. Also there is a canonical surjective mapping from $\gamma^{\omega}$ onto $[\gamma]^{\aleph_{0}}$. If for some $\gamma<\boldsymbol{\Theta}$ there is an unbounded mapping from $[\gamma]^{\aleph_{0}}$ to $\boldsymbol{\theta}$, 
since $\Theta$ is regular, there is an onto mapping from $[\gamma]^{\aleph_{0}}$ to $\Theta$. This would be a contradiction.

Now we define our sequence by induction on $\alpha<\omega_{1}$.

$\gamma_{0}=\omega$.

For $\alpha<\omega_{1}$, inductively assume that $\gamma_{\xi}$ for $\xi<\alpha$ are defined, satisfying that if $y \in\left[\gamma_{\xi}\right]^{\aleph_{0}}$, and if $\gamma>\gamma_{\xi+1}$ then there is a $\rho$ such that $\gamma_{\xi} \leq \rho<\gamma_{\xi+1}$ and $f_{\rho}\left\lceil y=f_{\gamma}\lceil y\right.$.

If $\alpha$ is a limit, then $\gamma_{\alpha}=\sup \left\{\gamma_{\xi} \mid \xi<\alpha\right\}$.

If $\alpha=\xi+1$, then we define a function $\beta_{\xi}:\left[\gamma_{\xi}\right]^{\aleph_{0}} \rightarrow \Theta$ by $\gamma_{\xi}<\beta_{\xi}(x)<\boldsymbol{\theta}$ is the least $\beta$ such that

$$
\forall \gamma<\boldsymbol{\theta} \gamma>\beta \Rightarrow \exists \rho\left(\gamma_{\xi} \leq \rho<\beta \wedge f_{\rho}\left\lceil x=f_{\gamma}\lceil x) .\right.\right.
$$

Then define $\gamma_{\alpha}$ to be the least $\lambda<\Theta$ such that $\beta_{\xi}:\left[\gamma_{\xi}\right]^{\aleph_{0}} \rightarrow \lambda$.

This finishes the definition of the sequence with the desired properties.

Let $\eta=\sup \left\{\gamma_{\xi} \mid \xi<\omega_{1}\right\}$.

We now define a one-to-one sequence $\left\langle x_{\alpha} \mid \alpha<\omega_{1}\right\rangle$ of length $\omega_{1}$ from $\eta$ as follows.

$x_{n}=n$ for $n<\omega$.

For $\omega \leq \alpha<\omega_{1}$, assume that $\left\langle x_{\beta} \mid \beta<\alpha\right\rangle$ has been defined and each $x_{\beta}<\eta$. Let $A=\left\{x_{\beta} \mid \beta<\alpha\right\}$. Let $\xi$ be the least such that $A \in\left[\gamma_{\xi}\right]^{\aleph_{0}}$. Let $\rho$ be the least such that $\gamma_{\xi} \leq \rho<\gamma_{\xi+1}$ and $f_{\rho}\left|A=f_{\eta}\right| A$.

Then define $x_{\alpha}=\rho$.

Let $X=\left\{x_{\alpha} \mid \omega \leq \alpha<\omega_{1}\right\}$.

Define $h: X \rightarrow \omega$ by $h(\alpha)=f_{\eta}(\alpha)$. Let $H \subseteq X$ be uncountable such that $h \backslash H$ is a constant. Now if $\omega \leq \alpha<\beta<\omega_{1}$, if $x_{\alpha}, x_{\beta} \in H$, then $f\left(\left\{x_{\alpha}, x_{\beta}\right\}\right)=f_{x_{\beta}}\left(x_{\alpha}\right)=f_{\eta}\left(x_{\alpha}\right)=h\left(x_{\alpha}\right)=a$. Here $a$ is the value of $h$ on $H$. Hence $H$ is homogeneous for $f$. (Actually $H \cup\{\eta\}$ is homogeneous for $f$.)

This finishes the proof.

We now present an alternative proof using a pressing-down argument suggested by $\mathrm{S}$. Todorcevic. What we actually prove by this method is the following slightly stronger form: If $\boldsymbol{\Theta}$ is regular, $\boldsymbol{\Theta} \rightarrow\left(\boldsymbol{\Theta},\left(\omega_{1}+1\right)_{\omega}\right)^{2}$, i.e., for each $f:[\Theta]^{2} \rightarrow \omega$, either there is an unbounded subset $H \subseteq \boldsymbol{\Theta}$ such that $f^{\prime \prime}[H]^{2}=\{0\}$, or for some $i>0$ there is a subset $H \subseteq \Theta$ of order type $\omega_{1}+1$ such that $f^{\prime \prime}[H]^{2}=\{i\}$.

As above, let $g:[\Theta]^{2} \rightarrow \omega$ be given. By redefining the function if necessary we may assume that for each $\alpha<\Theta$ we have $g(\{0, \alpha\})=0$.

Let $\tau=\omega-\{0\}$.

For each $\delta$ of cofinality $>\omega$, we define a sequence $f_{\delta}: \tau \times \omega_{1} \rightarrow \delta$ as follows:

Fix $i \in \tau$.

$f_{\delta}(\langle i, 0\rangle)=$ the least $\alpha<\delta$ so that $g(\{\alpha, \delta\})=i$ if there is such. Otherwise let $f_{\delta}(\langle i, 0\rangle)=0$.

Inductively, let $\gamma<\omega_{1}$. Define $f_{\delta}(\langle i, \gamma\rangle)=$ the least $\alpha<\delta$ so that $g(\{\alpha, \delta\})=i$ and for all $\eta<\gamma \quad f_{\delta}(\langle i, \eta\rangle)<\alpha$ and $g\left(\left\{f_{\delta}(\langle i, \eta\rangle), \alpha\right\}\right)=i$, if there is such. Otherwise, $f_{\delta}(\langle i, \gamma\rangle)=0$.

Case 1. There is some $i \in \tau$ and some $\delta$ whose cofinality is larger than $\omega$ and for every $\gamma<\omega_{1}$ we have $f_{\delta}(\langle i, \gamma\rangle)>0$. 
Then we have a homogeneous set $H$ of order type $\omega_{1}+1$ for $g$ so that $g^{\prime \prime}[H]^{2}=\{i\}$ for some $i>0$.

\section{Case 2. Otherwise.}

In this case we show that there is an unbounded subset of $\Theta$ which is homogeneous for $g$ with color 0 .

By Fodor's lemma, we can have a stationary subset $S \subseteq \boldsymbol{\theta}$ so that for each $\delta \in S, c f(\delta)>\omega$, and for some $\eta<\omega_{1}$ and for some $\alpha<\theta$ we have if $\delta \in S$ then $\eta$ is the least so that $f_{\delta}(\langle i, \eta\rangle)=0$ for all $i \in \tau$ and $\alpha=$ $\sup \left\{f_{\delta}(\langle i, \gamma\rangle)+1 \mid \gamma<\eta, i \in \tau\right\}$.

Now consider the function $G: S \rightarrow \alpha^{\tau \times \eta}$ defined by $G(\delta)=f_{\delta} \mid \tau \times \eta$.

If there is some $h \in \alpha^{\tau \times \eta}$ so that there are unboundedly many $\delta \in S$ so that $G(\delta)=h$, then these $\delta$ 's constitute our 0-homogeneous set.

So assume otherwise. Then $G$ is bounded-to-one. Hence we can have an unbounded subset $A \subseteq S$ so that $G \backslash A$ is one-to-one. But this means that there is a surjective function from $\alpha^{\tau \times \eta}$ onto $\Theta$, which in turn implies that there is a surjective mapping from the reals to $\Theta$. This is a contradiction.

This finishes the proof.

\section{REFERENCES}

1. U. Abraham, M. Rubin, and S. Shelah, On the consistency of some partition theorems for continuous coloring, and the structure of $\aleph_{1}$-dense real order types, Ann. Pure Appl. Logic 29 (1985), 123-206.

2. J. Baumgartner, Applications of the proper forcing axiom, Handbook of Set Theoretic Topology (K. Kunen and J. Vaughan, eds.), Elsevier, 1984, pp. 913-959.

3. A. Blass, A partition theorem for perfect sets, Proc. Amer. Math. Soc. 82 (1981), 271-277.

4. M. Davis, Infinite games of perfect information, Advances in Game Theory, Ann. of Math. Studies, no. 52, Princeton Univ. Press, 1964, pp. 85-101.

5. A. Dodd and R. Jensen, The core model, Ann. Math. Logic 20 (1981), 43-75.

6. P. Erdös and R. Rado. A partition calculus in set theory, Bull. Amer. Math. Soc. 62 (1956), 427-489.

7. F. Filipczak, Sur les fonctions continues relativement monotones, Fund. Math. 58 (1966), 75-87.

8. F. Galvin, Partition theorems for the real line, Notices Amer. Math. Soc. 15 (1968), 660.

9. L. Harrington, M. Marker, and S. Shelah, Borel orderings, Trans. Amer. Math. Soc. 310 (1988), 293-302.

10. T. Jech, Set theory, Academic Press, 1978.

11. H. D. Donder, R. Jensen, and B. Koppelberg, Some applications of the core model, Set Theory and Model Theory, Lecture Notes in Math., vol. 872, Springer-Verlag, 1981, pp. 55-97.

12. R. Mansfield, Perfect subsets of definable sets of real numbers, Pacific J. Math. 35 (1970), 451-457.

13. D. Martin and J. Steel, Projective determinacy, Proc. Nat. Acad. Sci. U.S.A. 851 (1988), 6587-6591.

14. __ A proof of projective determinacy, J. Amer. Math. Soc. 2 (1989), 71-125.

15. J. Mycielski, Independent sets in topological algebras, Fund. Math. 55 (1964), 139-147.

16. F. Ramsey, On a problem of formal logic, Proc. London Math. Soc. 30 (1930), 264-286.

17. W. Sierpiński, Sur un probleme de la théorie des relations, Ann. Scuola Norm. Sup. Pisa 2 (1937), 285-287. 
18. J. Shoenfield, The problem of predicativity, Essays on the Foundations of Mathematics (Y. Bar-Hillel, E. Poznanski, M. O. Rabin, and A. Robinson, eds.), Magnes Press, Jerusalem, 1961, pp. 132-139.

19. R. Solovay, On the cardinality of $\Sigma_{2}^{1}$ sets of reals, Foundations of Mathematics, Sympos. Commemorating Kurt Gödel (Buloff, ed.), Springer-Verlag, 1969, pp. 58-73.

20. S. Todorcevic, Partition problems in topology, Contemp. Math., vol. 84, Amer. Math. Soc., Providence, R.I., 1989.

21. __ Two examples of Borel partially ordered sets with countable chain conditions, preprint, 1990.

22. __ Conference talk, MSRI, October 1989.

23. B. Velickovic, Conference talk, MSRI, October 1989.

24. H. Woodin, Supercompact cardinals, sets of reals, and weakly homogeneous trees, Proc. Nat. Acad. Sci. U.S.A. 85 (1988), 6587-6591.

25. P. Erdös, K. Kunen, and R. Mauldin, Some additive properties of sets of real numbers, Fund. Math. (1981), 187-199.

26. D. Gale and F. Stewart, Infinite games with perfect information, Contributions to the Theory of Games. II, Ann. of Math. Studies, no. 281, Princeton Univ. Press, 1953, pp. 245-266.

27. R. Solovay, A model of set theory in which every set of reals is Lebesgue measurable, Ann. of Math. (2) (1970), 1-56.

28. A. Miller, Special subsets of the real line, Handbook of Set Theoretic Topology (K. Kunen and J. Vaughan, eds.), Elsevier, 1984, pp. 201-233.

29. R. Solovay and S. Tennenbaum, Iterated Cohen extensions and Souslin's problem, Ann. of Math. (2) 4 (1971), 201-245.

30. D. A. Martin, Borel determinacy, Ann. of Math. (2) 102 (1975), 363-371.

Department of Mathematics, National University of Singapore, 10 Kent Ridge CresCENT, SINGAPORE 0511 